\title{
Glioblastoma exosomes influence glial cell hyaluronic acid deposition to promote invasiveness
}

\section{Dominik Koessinger ${ }^{1,2,3,7}$, David Novo ${ }^{1,4,7}$, Anna Koessinger ${ }^{1,2}$, Désirée Zerbst ${ }^{1,2}$, Madeleine Moore ${ }^{1}$, Louise Mitchell ${ }^{1}$, Matthew Neilson ${ }^{1}$, Katrina Stevenson ${ }^{2}$, Anthony Chalmers $^{2}$, Joanna Birch ${ }^{2} \&$ Jim Norman ${ }^{1,2,6}$}

${ }^{1}$ Beatson Institute for Cancer Research, Glasgow, Scotland. G61 1BD

${ }^{2}$ Institute of Cancer Sciences, University of Glasgow, Glasgow G61 1QH, U.K

${ }^{3}$ Current address: Department of Neurosurgery, Freiburg University Hospital, Breisacher Str.

64, 79106 Freiburg, Germany

${ }^{4}$ Current address: Francis Crick Institute, 1 Midland Road, London NW1 1ST

${ }^{6}$ Lead contact author

${ }^{7}$ Shared first authorship

Address for correspondence; j.norman@beatson.gla.ac.uk

Word count: 5878 (excluding summary, methods, references, and supplement)

Short title: Glioma EVs foster a pro-invasive microenvironment

Keywords: extracellular vesicles, exosomes, GBM, cell migration, invasion, astrocytes, extracellular matrix, hyaluronic acid, mutant p53, 


\section{SUMMARY (129 words)}

A deeper understanding of the mechanisms invoked by glioblastoma (GBM) to infiltrate the brain is needed to develop approaches to contain the disease and reduce recurrence. Here we report that GBM cells which have acquired a p53 mutation ( $\mathrm{p} 53^{273 \mathrm{H}}$ ) with established proinvasive gain-of-function release exosomes/extracellular vesicles (EVs) containing a sialomucin, podocalyxin (PODXL), which encourages other brain cells, such as astrocytes to deposit extracellular matrix (ECM) with increased levels of hyaluronic acid (HA). This HA-rich ECM, in turn, promotes invasiveness of the GBM cells. Consistently, CRISPR-mediated deletion of PODXL opposes infiltration of GBM in vivo. This study offers a mechanistic basis for the particularly poor prognosis of GBM which are driven by the $\mathrm{p} 53^{273 \mathrm{H}}$ mutant, and how these tumours influence the wider brain ECM microenvironment through which glioma cells invade. 
bioRxiv preprint doi: https://doi.org/10.1101/2022.02.11.480036; this version posted February 11, 2022. The copyright holder for this preprint (which was not certified by peer review) is the author/funder. All rights reserved. No reuse allowed without permission.

Glioma EVs foster a pro-invasive microenvironment

Koessinger, Novo et al.

\section{INTRODUCTION}

Glioblastoma (GBM) exhibit highly invasive/infiltrative behaviour which is largely responsible for the intractable nature of the disease (Miller \& Perry, 2007). At diagnosis GBM usually display widespread infiltration of the surrounding brain tissue which precludes in toto resection and focussed radiotherapy and, therefore, leads to relapse. Furthermore, widespread insinuation of invasive cells into brain tissue is responsible for many of the neurological and cognitive symptoms which contribute to GBM's morbidity (Drumm, Dixit et al., 2020). Thus, an understanding of the mechanisms though which GBM acquire infiltrative/invasive characteristics, and the cellular mechanisms involved in this, is necessary to assist development of pharmacological strategies to control the disease following resection both before and following chemo and radiotherapy - thus improving accessibility for treatment in the case of relapse and ameliorating its associated neurological and cognitive dysfunction.

Carcinomas acquire metastatic characteristics through cell autonomous mechanisms, such as the influence of oncogenes on the cancer cell's migratory machinery, but also by influencing the microenvironment. This can occur both locally and at some distance from the primary tumour. Mechanisms through which cancer secretomes communicate with other cells to influence local extracellular matrix (ECM), metabolic and immune microenvironments are well-established (Padua \& Massague, 2009). Furthermore, the ways in which cancers influence the microenvironment in distant organs to prime these for subsequent metastatic colonisation are now becoming clear. Soluble components of the cancer secretome - such as GM-CSF - can mobilise myeloid populations (such as macrophages and neutrophils) to metastatic target organs, and therein generate immunosuppressive microenvironments conducive to metastatic seeding (Kitamura, Qian et al., 2015). Additionally, tumour-derived extracellular vesicles (EVs) - particularly exosomes, which are lipid bilayer-bounded vesicles of approximately $80-150 \mathrm{~nm}$ diameter - generate invasive microenvironments and metastatic niches (Wortzel, Dror et al., 2019). This is mediated by the ability of EVs to alter the recruitment or phenotype of immune cells within metastatic niches and these changes have been reported to involve alterations to the vasculature and recruitment of myeloid cell populations, such as macrophages which have assumed immunosuppressive phenotypes. Furthermore, evidence is accumulating that tumour EVs can alter the ECM (for instance, by increasing fibronectin deposition or altering collagen organisation) within organs such as the liver and lung to generate a pro-invasive microenvironment, thus favouring metastatic seeding. (Costa-Silva, Aiello et al., 2015, Novo, Heath et al., 2018)

Infiltrative behaviour in GBM can be mediated via cell autonomous mechanisms and, more recently, this has been shown to involve more complex intercellular communication 
processes leading to alterations in the nearby and distant brain microenvironment. GBM cells can communicate with other glioma cells or non-malignant ones, such as astrocytes, oligodendrocytes, microglia, or endothelia in a way that favours infiltration of the brain (Broekman, Maas et al., 2018). Invading glioma cells produce a range of protrusion-types including tumour microtubes and the thinner tunnelling nanotubes - to form functional intercellular communication networks which alter the local and wider brain microenvironment - which favours infiltration and therapy resistance (Osswald, Jung et al., 2015, Pinto, Saenzde-Santa-Maria et al., 2021). EVs are also emerging as mediators of communication between GBM cells and the brain microenvironment. Indeed, numerous studies have highlighted the potential for GBM EVs to influence other cells in the brain in a way that would be expected to favour tumour cell proliferation, angiogenesis and immune infiltration (Treps, Perret et al., 2017, Zappulli, Friis et al., 2016). As with metastatic priming, a number of studies now indicate that GBM EVs increase recruitment of myeloid cell populations with tumour promoting and/or immunosuppressive properties (de Vrij, Maas et al., 2015, Domenis, Cesselli et al., 2017, Gabrusiewicz, Li et al., 2018, Hellwinkel, Redzic et al., 2016). However, despite the clear importance of the brain ECM to the infiltrative microenvironment, there is little information as to how GBM EVs might alter the brain ECM, and what the role of the brain's main ECM depositing cell-type, the astrocyte, might have this regard. Therefore, we have studied how an oncogene - the $\mathrm{p} 53^{273 \mathrm{H}}$ mutant - which is closely associated with poor clinical outcomes, alters the constitution of EVs released by patient-derived glioma cells. We report that EVs released by $\mathrm{p} 53^{273 \mathrm{H}}$-expressing GBM cells are responsible for enabling their invasive behaviour in vivo, and this is due to the ability of these EVs to influence the hyaluronic acid (HA) content of ECM deposited by astrocytes. 
bioRxiv preprint doi: https://doi.org/10.1101/2022.02.11.480036; this version posted February 11, 2022. The copyright holder for this preprint (which was not certified by peer review) is the author/funder. All rights reserved. No reuse allowed without permission.

Glioma EVs foster a pro-invasive microenvironment

Koessinger, Novo et al.

\section{RESULTS}

\section{EVs from infiltrative GBM cells foster a pro-migratory microenvironment in brain slices}

We deployed two primary patient-derived glioma stem-like cell (GSC) lines, G7 and E2, which are derived from resected patient tissue as previously described (Ahmed, Carruthers et al., 2015, Fael Al-Mayhani, Ball et al., 2009). These lines were chosen in view of the markedly different invasive/infiltrative characteristics that they display in vivo. Indeed, using bioluminescence and other approaches we have recently shown that G7 GSC grow as a solid tumour with moderately invasive margins, whereas E2 disseminate throughout the brain as widely scattered tumour cells (Koessinger, Koessinger et al., 2020). To determine whether this was owing to their intrinsically different migratory behaviour in a neuronal microenvironment, we plated GFP-expressing G7 and E2 cells onto brain slices from 5-8week Cl57/BI6 mice and recorded their movement using fluorescence time-lapse microscopy. GBM cells migrate throughout the brain preferably alongside white matter tracts, or within the perivascular niche (Manini, Caponnetto et al., 2018). Therefore, to minimise any bias that might result from inadvertent focus on white matter track-rich regions we tracked $\geq 60$ cells per condition across the entire brain slice. Surprisingly, both the G7 and E2 cell lines migrated similarly (and poorly) on brain slices (Figure S1A) despite their markedly different invasive behaviour in vivo (Koessinger et al., 2020). We hypothesised that factors released by the GBM cells may induce changes in the brain microenvironment which promote tumour cell invasion, and that this may take some time to establish. EVs are emerging as a component of the tumour secretome with key roles in influencing the microenvironment both locally and at distance from the primary tumour. We used differential centrifugation to purify EVs from GSC-exposed medium and analysed these using nanoparticle tracking, which indicated that both G7 and E2 cells released EVs in similar quantities and with indistinguishable size distributions (Figure S1B). We then incubated brain slices with equal quantities of EVs from G7 or E2 cells for $72 \mathrm{hr}$. GFP-expressing E2 cells were subsequently plated onto the brain slices, and we measured their migration. This indicated that pre-treatment of brain slices with EVs from E2 cells significantly increased the speed of subsequently plated GBM cells, whilst EVs from G7 cells were ineffective in this regard (Figure $1 \mathrm{~A}$ ). Consistent with this enhancement of their migratory behaviour, GBM cells plated onto brain slices pre-treated with EVs from E2 cells displayed a more invasive phenotype characterised by extension of processes and invasive protrusions which resulted in alterations to the cell envelope - as evidenced by reduced cell roundness/symmetry (Figure 1B). Conversely, when GBM cells were plated directly onto plastic dishes their migration speed was unaffected by pre-treatment for $72 \mathrm{hr}$ with EVs (Figure S1C), indicating 
bioRxiv preprint doi: https://doi.org/10.1101/2022.02.11.480036; this version posted February 11, 2022. The copyright holder for this preprint (which was not certified by peer review) is the author/funder. All rights reserved. No reuse allowed without permission.

Glioma EVs foster a pro-invasive microenvironment

Koessinger, Novo et al.

that the pro-migratory effects of EVs were mediated by influencing the microenvironment of the brain slices and not via direct effects on the GBM cells.

We have previously demonstrated that the ability of EVs from primary tumours to generate pro-invasive microenvironments requires activity of the lipid kinase, diacylglycerol kinase- $\alpha$ (DGKa) in the 'recipient' cells which mount the response to the EVs (Novo et al., 2018). We, therefore, deployed DGKa knockout mice $\left(\mathrm{DGKa}^{-1}\right)$ to determine whether this kinase is required for a 'recipient' brain cell population to generate a pro-migratory microenvironment in response to GBM EVs. Brain slices from 5-8 week DGKa ${ }^{-1-} \mathrm{Cl} 57 / \mathrm{BI} 6$ mice were treated with EVs from E2 cells for $72 \mathrm{hr}$, and GFP-expressing E2 cells were subsequently plated onto these. GBM cells migrated poorly on brain slices from DGKa ${ }^{-/-}$mice (as they did on slices from $\mathrm{DGK}^{+/+}$mice), but this was unaffected by pre-treatment with EVs from E2 cells, indicating that expression of DGKa is required for a recipient brain cell population to generate a pro-invasive/migratory microenvironment in response to GBM EVs (Figure 1A).

\section{EVs from infiltrative GBM cells encourage astrocytes to deposit pro-migratory ECM.}

Astrocytes, the most abundant cells in the brain are well-positioned to influence the brain microenvironment in response to EVs from GBM. Indeed, a principal tasks of astrocytes is to deposit ECM in the brain and thus maintain its appropriate microenvironment. To determine whether EVs from GBM cells can influence the nature of the astrocyte-deposited ECM, we incubated astrocytes with EVs from E2 or G7 cells for 72 hours. We then re-plated the astrocytes at high confluence and allowed them to deposit ECM for 6 days. Astrocyte cultures were then de-cellularised and migration of GBM cells (either G7 or E2 cells) plated onto these was determined using time-lapse microscopy. This indicated that pre-treatment of astrocytes with EVs from E2 cells prior to ECM generation significantly increased the speed of both E2 and G7 GBM cells plated on these matrices, whereas pre-treatment with EVs from G7 cells was ineffective in this regard (Figure 1C). Moreover, following treatment with a DGKa inhibitor (R59022) (de Chaffoy de Courcelles, Roevens et al., 1985, Rainero, Caswell et al., 2012) - the inhibitor being added during exposure to EVs and maintained during the 6day ECM-depositing period - astrocytes deposited ECM with limited ability to support GBM cell migration irrespective of whether or not they were pre-treated with EVs from E2 cells. These data indicate that EVs from the highly infiltrative E2 GBM cells educate astrocytes to deposit ECM with increased capacity to support GBM cell migration, and that the activity of DGKa is required for astrocytes to do this.

\section{Deletion of $\mathrm{p53}^{273 \mathrm{H}}$ reduces the ability of EVs from GBM cells to foster a pro-migratory microenvironment}


bioRxiv preprint doi: https://doi.org/10.1101/2022.02.11.480036; this version posted February 11, 2022. The copyright holder for this preprint (which was not certified by peer review) is the author/funder. All rights reserved. No reuse allowed without permission.

Glioma EVs foster a pro-invasive microenvironment

Koessinger, Novo et al.

The impact of p53 on tumour invasiveness has previously been linked to certain gain-offunction point mutations (Muller, Caswell et al., 2009). We, therefore, examined the p53 reading frame in E2 and G7 cell lines from deep-sequencing data and compared these with the International Agency for Research on Cancer (IARC) database. Our analysis revealed that the highly infiltrative $\mathrm{E} 2$ cells express the $\mathrm{p} 53^{\mathrm{R} 273 \mathrm{H}}$ mutant $(83 \%$ of reads), which has well-characterised pro-invasive/metastatic gain-of-function properties (Muller et al., 2009, Olive, Tuveson et al., 2004, Powell, Piwnica-Worms et al., 2014, Weissmueller, Manchado et al., 2014) (Supplementary table 1a). On the other hand, expression of mutated p53s in the less invasive $\mathrm{G} 7$ cells is less consistent, with reads being detected for various mutations (p.R248Q p.R282W p72r, each approx. 50\% of reads) (Supplementary table S1b). The $\mathrm{R} 248 \mathrm{Q}$ mutation has been linked to increased tumour growth but not metastasis, and the role of $p 53^{282 \mathrm{~W}}$ in tumour progression is not established (Ahn, Kim et al., 2017, SchulzHeddergott, Stark et al., 2018).

To determine whether expression of $\mathrm{p} 53^{273 \mathrm{H}}$ contributes to the ability of E2 cells to foster (via EV release) a microenvironment conducive to invasiveness, we generated clones of E2 cells in which the gene for p53 was targeted using two independent CRISPR guide sequences. We used Western blotting to confirm deletion of $\mathrm{p} 53^{273 \mathrm{H}}$, and to ensure that expression of neuronal stem cell markers, Nestin and SOX2, had been maintained during the gene deletion and cell selection procedure (Figure 2A). Deletion of mutant p53 did not alter the rate at which $\mathrm{E} 2$ cells proliferated (Figure $2 \mathrm{~B}$ ). Moreover, the quantity and size-distribution of EVs purified from conditioned medium from p53 knockout (p53-KO) E2 cells did not differ from the corresponding CRISPR-ctrl E2 cells, indicating that mutp53 ${ }^{\mathrm{R} 273 \mathrm{H}}$ does not influence the number of EVs released from GBM cells (Figure. $2 \mathrm{C}$ ). As previous work indicates that mutant p53 generates pro-invasive niches by modulating the quantity of the sialomucin, podocalyxin (PODXL) that is sorted into EVs (Novo et al., 2018), we determined the

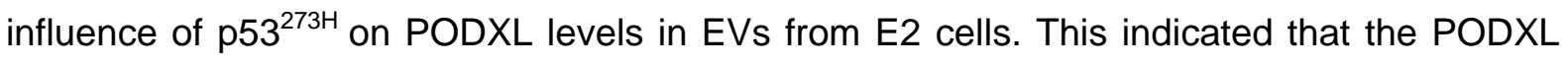
levels in EVs were increased by deletion of mutant p53 ${ }^{273 H}$ from E2 cells (Figure 2D); an observation consistent with previous findings in carcinoma cell lines, and which moots the probability that EVs from p53-KO E2 cells might have altered ability to foster pro-invasive microenvironments (Novo et al., 2018). Indeed, EVs from p53-KO E2 cells displayed reduced capacity to influence astrocyte ECM deposition in a way that supported migration of GBM cells (Figure 2E).

\section{PODXL content of GBM EVs is critical to influencing astrocyte ECM deposition}

The PODXL content of carcinoma cell EVs must be within a certain range for them to encourage fibroblasts to deposit pro-migratory ECM, and expression of mutant p53 functions to move EV PODXL levels into this range (Novo et al., 2018). To determine whether PODXL 
bioRxiv preprint doi: https://doi.org/10.1101/2022.02.11.480036; this version posted February 11, 2022. The copyright holder for this preprint (which was not certified by peer review) is the author/funder. All rights reserved. No reuse allowed without permission.

Glioma EVs foster a pro-invasive microenvironment

Koessinger, Novo et al.

levels in EVs from GBM is also causally linked to their ability to alter ECM deposition by astrocytes, we generated E2 cells in which PODXL levels were either increased (by overexpression of GFP-tagged PODXL) or reduced (using CRISPR gene deletion). Furthermore, because Rab35 controls PODXL trafficking to EVs - and deletion of this GTPase opposes the ability of EVs from carcinoma cells to influence ECM deposition (Novo et al., 2018) - we also used CRISPR to generate Rab35 knockout GBM cells. Immunoblotting confirmed that EV PODXL levels were respectively increased or decreased by PODXL over-expression or knockout (Figure 3A, B) and, consistent with its role in PODXL sorting, deletion of Rab35 reduced EV PODXL levels (Figure $3 C$ ). These manipulations of PODXL and/or Rab35 levels did not alter GBM cell proliferation nor expression of neuronal stem cell markers (Figure 3AC). We then pre-treated astrocytes with EVs from PODXL over-expressing or -deficient, and Rab35 knockout E2 cells for $72 \mathrm{~h}$, and allowed them to deposit ECM for a further 5 days. Astrocyte-derived ECM was de-cellularised, and migration of GBM cells on these ECMs determined using time-lapse microscopy and cell tracking. This indicated that that EVs from PODXL-GFP overexpressing (Figure 4A) and from PODXL (Figure 4B) or Rab35 (Figure 4C) knockout E2 cells were unable to influence astrocyte ECM deposition in a way that supported increased migration of GBM cells. Finally, we pre-treated mouse brain slices with EVs from control and PODXL or Rab35 knockout E2 cells and subsequently plated GBM cells onto these. This indicated that knockout of PODXL or Rab35 opposed the ability of EVs from GBM cells to foster pro-migratory microenvironments in the brain (Figure 4C). Taken together, these data indicate that expression of $p 53^{273 H}$ in GBM cells fosters a pro-invasive brain microenvironment, and that this is via regulation of EV PODXL content and the influence of this on the ECM depositing capacity of astrocytes.

\section{EVs promote GBM cell migration by influencing the hyaluronic acid content of astrocyte ECM}

EVs from mutant p53-expressing carcinoma cells generate pro-invasive niches in metastatic target organs (such as the lung), by influencing deposition of fibrillar ECM components, such as fibronectin and collagen (Novo et al., 2018). However, brain ECM is composed primarily of proteo- and glycosamino-glycans and is largely devoid of fibrillar proteins (Ruoslahti, 1996). We, therefore, used a panel of lectins and other reagents recognising carbohydrate moieties (such as hyaluronic acid-binding protein (HABP) and anti-chondroitin sulphate) to screen for EV-driven alterations to glycan/polysaccharide species in ECM deposited by astrocytes. Most carbohydrate species were not detectably different between the ECM deposited by naïve and EV-educated astrocytes (not shown). However, pre-treatment of astrocytes with EVs from E2 cells drove a significant increase in the hyaluronic acid $(\mathrm{HA})$ (detected by HABP) (Figure $5 \mathrm{~A}$ ), and a moderate, but not significant, decrease the 
bioRxiv preprint doi: https://doi.org/10.1101/2022.02.11.480036; this version posted February 11, 2022. The copyright holder for this preprint (which was not certified by peer review) is the author/funder. All rights reserved. No reuse allowed without permission.

Glioma EVs foster a pro-invasive microenvironment

Koessinger, Novo et al.

chondroitin sulphate (CS) (Figure S2A) content of the ECM they deposited. Conversely, pretreatment of astrocytes with EVs from the less invasive G7 GBM cells, or EVs from E2 cells in which either mutant $\mathrm{p} 53^{273 H}$, PODXL or Rab35 had been deleted using CRISPR, were ineffective in this regard (Figure $5 \mathrm{~B}$ ). HA has a well-established role in promoting migratory and invasive behaviour of many cell types (Park, Kwak et al., 2008) - including tumour cells - and examination of Z-projections of 3D confocal image stacks indicated that EVs from E2 cells most significantly increased the HA content of the ECM on upper face of the astrocyte cultures where it is ideally placed to interact with GBM cells plated onto it (Figure S2B). Moreover, the HA present on the upper face of the astrocyte cultures in organised into long cable-like structures likely to influence migration of interacting cells (Fig. 5A). We, therefore, allowed astrocytes to deposit ECM in the presence of hyaluronidase (Hase), an enzyme which catalyses the hydrolysis of $\mathrm{HA}$, and this led to the deposition of ECM with reduced $\mathrm{HA}$ content (Figure 5C). We next plated GBM cells onto ECM generated in the presence and absence of Hase. This indicated that the ability of EV-pre-treated astrocytes to generate ECM which supports GBM cell migration was completely ablated by Hase treatment, whilst Hase did not alter the (limited) ability of ECM deposited by EV-naïve astrocytes to support GBM cell migration (Figure 5D).

Taken together these data indicate that GBM expressing the $\mathrm{p} 53^{273 \mathrm{H}}$ mutant release EVs which, by virtue of their PODXL content, encourage astrocytes to deposit ECM with increased HA content which, in turn, promotes GBM cell migration.

\section{PODXL-expression is required for mutant $\mathrm{p} 53^{273 \mathrm{H}}$-driven infiltrative behaviour of GBM in vivo}

Having established three key components - mutant p53 ${ }^{273 H}$, PODXL and Rab35 - necessary for release of EVs which control astrocyte ECM deposition, and described mechanistic details of how they achieve this, we wished to determine whether these components influence GBM infiltration and invasiveness in vivo. We injected mutant p53, PODXL or Rab35 knockout E2 cells (or CRISPR-control) into the right striatum of CD1 nude mice. 9 weeks after injection the number of (proliferating) tumour cells in coronal brain sections (2 consecutive, $50 \mu \mathrm{m}$ sections per mouse) was quantified by Ki67-staining followed by automated cell counting. Indeed, Ki67 staining clearly identifies the nuclei of tumour cells in a way that enables automated analysis. However, Ki67 staining may, due to its inability to detect non-proliferating cells, underestimate the number of invading tumour cells. We, therefore, confirmed that results from Ki67 and human-specific Leukocyte Antigen (to identify the human glioma cells) staining correlated closely (Birch, Strathdee et al., 2018). Moreover, automated counting was undertaken using parameters which excluded Ki67-positive resident brain cells in the subventricular zone. Knockout of mutant p53 or Rab35 reduced 
tumour growth to an extent which precluded us from determining how these potentially proinvasive factors might influence infiltration (Figure S3). However, as the total number of Ki67-positive GBM cells in the brain was not significantly altered by PODXL knockout (as determined by quantification of either 2 or 5 consecutive $50 \mu \mathrm{m}$ sections per mouse) (Figure S3), we were able to determine how PODXL expression influenced the proportion of GBM cells that had migrated from the ipsilateral to the contralateral (left) hemisphere. This revealed that loss of PODXL significantly reduced the proportion of Ki67-positive GBM cells that had moved from the left to the right side of the brain, indicating that PODXL expression contributes to efficient long-range infiltrative/migration of GBM cells (Figure 6A, B). 
bioRxiv preprint doi: https://doi.org/10.1101/2022.02.11.480036; this version posted February 11, 2022. The copyright holder for this preprint (which was not certified by peer review) is the author/funder. All rights reserved. No reuse allowed without permission.

Glioma EVs foster a pro-invasive microenvironment

Koessinger, Novo et al.

\section{DISCUSSION}

This study has identified a means of EV-mediated intercellular communication in which a specific oncogene enables glioblastomas to reprogramme ECM deposition by astrocytes to promote cancer infiltration throughout the brain. This proceeds via three sequential and distinct mechanistic events as summarised in Fig. 7: 1. A gain-of-function p53 mutation (p53 ${ }^{273 H}$ ), which is common in GBM, acting in combination with a GTPase of the Rab family (Rab35) to control sorting of PODXL into EVs; 2. PODXL-containing EVs from GBM cells encouraging astrocytes to deposit ECM which is particularly rich in HA, and; 3 . HA-rich extracellular ECM, in turn, encouraging GBM cells to be more invasive and to migrate long distances to infiltrate the brain (Fig. 7). Furthermore, expression of DGKa by astrocytes is required for them to deposit pro-migratory ECM in response to PODXL-containing EVs.

Metastatic spread depends, not only on the inherent migratory capabilities of cancer cells, but also on their ability to generate microenvironments permissive to cell migration, invasion, and colonisation. Communication between cancer cells in the primary tumour and ECMdepositing fibroblasts in metastatic niches is central to this process (Costa-Silva et al., 2015, Novo et al., 2018) and, in this study, we demonstrate that a process analogous to metastatic niche priming may occur as GBM infiltrate the brain. Indeed, patient-derived glioma cells can display markedly different migratory behaviour within the brain microenvironment, but this owes less to their intrinsic migratory capacity than it does to their influence on ECM deposition by glial cells. Principal amongst the similarities between this mechanism of glioma infiltration and metastatic niche priming by carcinomas is reliance on an EV-transduced signal emanating from the primary tumour cell which influences the behaviour of ECMdepositing cells. This signal is switched-on in both carcinoma and GBM cells by acquisition of a particular p53 mutation - p53 $3^{273 H}$ - which acts in combination with Rab35 to tune the PODXL content of tumour cell EVs into a range that influences ECM deposition by recipient cells - be they astrocytes or fibroblasts. The p53 pathway is deregulated in many GBM, and p53 itself is mutated in a way that might be expected to lead to loss of tumour suppressive function in most cell lines derived from GBM. Accordingly, p53 is mutated in both the G7 $\left(\mathrm{p} 53^{\mathrm{R} 248 \mathrm{Q}}\right.$ and $\mathrm{p} 53^{\mathrm{R} 282 \mathrm{~W}}$ ) and $\mathrm{E} 2\left(\mathrm{p} 53^{273 \mathrm{H}}\right)$ patient-derived cell lines, and $\mathrm{p} 53$ protein is wellexpressed in these two lines, consistent with the stabilising nature of these mutations (Figure $S 4 A$ ). However, given the novel gain-of-function that we here describe for $\mathrm{p} 53^{273 \mathrm{H}}$ - which enables E2 cells to release EVs capable of influencing astrocyte migration and ECM deposition - it is interesting to consider the impact of this p53 mutant on glioma progression. Low grade (I-III) glioma are largely uninvasive but, as the switch to grade IV GBM is accompanied by increased aggressiveness and invasiveness, we sought to determine

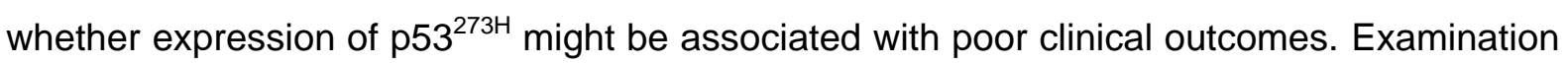


bioRxiv preprint doi: https://doi.org/10.1101/2022.02.11.480036; this version posted February 11, 2022. The copyright holder for this preprint (which was not certified by peer review) is the author/funder. All rights reserved. No reuse allowed without permission.

Glioma EVs foster a pro-invasive microenvironment

Koessinger, Novo et al.

of the merged Cell 2016 LGG and GBM dataset using cBioportal indicated that 40\% tumours (319/794) displayed mutations of the p53 gene, with the R273H locus being by far the most common p53 mutation - accounting for 22\% (69/319) of all p53 mutations (Figure S4B). However, the different prognosis of the glioma subtype represented within this dataset prevented us from drawing general conclusions regarding survival. As the glioma subtype with the highest rate of p53 mutation is IDH-mutant astrocytoma, we turned to the TCGA LGG PanCancer Atlas dataset (Ceccarelli, Barthel et al., 2016) to determine whether IDHmutant astrocytoma harbouring $\mathrm{p} 53^{273 \mathrm{H}}$ might progress more quickly than those bearing

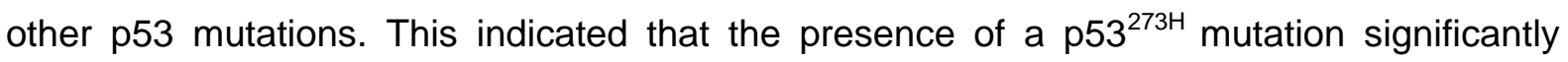
reduced overall median survival by comparison with tumours bearing other p53 mutations (Figure S4C). Thus, we submit that the ability of $p 53^{273 H}$ to influence the PODXL content of EVs and, thereby, engender a pro-invasive brain microenvironment, may contribute to the particularly fast progression and poor prognosis of gliomas bearing this p53 mutant.

Our data indicate that spread of both GBM and carcinoma cells which express $p 53^{273 H}$ is supported by release of EVs which instruct recipient cells (astrocytes and fibroblasts respectively) to deposit ECM with enhanced cell migration-supporting properties. Integral to the fibroblast response to PODXL-containing EVs is upregulation of integrin recycling which is dependent on DGKa - which operates by generating phosphatidic acid in the plasma membrane to enable docking and fusion of endocytic recycling vesicles (Rainero et al., 2012). Increased DGKa-dependent integrin recycling then changes fibroblast migratory behaviour - they move much more rapidly and erratically - which, in turn, leads to deposition of ECM with altered filament alignment which supports increased tumour cell migration (Novo et al., 2018). Unlike fibroblasts, astrocytes do not deposit ECM in which filamentalignment is detectable, but the HA content of astrocyte-deposited 'amorphous' ECM is markedly increased by glioma EVs, and it is this increased HA content which enables glioma cell migration. It is important, therefore, to consider how DGKa-mediated vesicle trafficking might influence HA deposition. HA synthases are transmembrane proteins which must be plasma membrane-localised to coordinate its export and incorporation into growing $\mathrm{HA}$ chains on the cell surface (Kobayashi, Chanmee et al., 2020). In fibroblasts, PODXLcontaining EVs promote plasma membrane delivery of numerous receptors - including integrins, receptor tyrosine kinases and the transferrin receptor - which cycle between endosomes and the cell surface (Novo et al., 2018). HA synthases also cycle between endomembrane compartments and the plasma membrane (Deen, Rilla et al., 2014), and it will be interesting to determine whether $\mathrm{EVs}$ from $\mathrm{p} 53^{273 \mathrm{H}}$-expressing glioma influence $\mathrm{HA}$ content of the ECM via increased cell delivery of these enzymes to the cell surface. Secondly, ECM-associated HA is turned-over by internalisation via endocytic (clathrin and 
non-clathrin dependent) and/or macropinocytic routes followed by hyaluronidase-mediated degradation in lysosomes (Kobayashi et al., 2020). As micropinocytosis and endocytosis/recycling are processes known to be influenced by DGKa (Broekman et al., 2018, Kovalenko, Sanin et al., 2021, Rainero et al., 2012), it will be interesting to determine whether EVs from glioma influence the rates of internalisation and degradation of HA.

Increased HA production by glioma has consistently been shown to be associated with processes driving GBM aggressiveness, such as enhanced cancer cell proliferation, chemoand radio-resistance, prevention of senescence and increased invasion of adjacent tissues (Pibuel, Poodts et al., 2021). Much of this can be attributed to engagement of receptors for $\mathrm{HA}$, such as CD44, on the surface of tumour cells. Indeed, CD44 has a well-established role in promoting invasive behaviour in many cancer-types (Jothy, 2003) and it is likely that the migratory response of GBM cells to HA-rich, astrocyte-deposited ECM is mediated via CD44. HA has anti-adhesive properties (Abatangelo, Vindigni et al., 2020), and these may contribute to increased cell migration. Interestingly, we have previously used AFM to show that exposure of fibroblasts to $\mathrm{EVs}$ from $\mathrm{mp} 53^{373 \mathrm{H}}$-expressing carcinomas markedly reduces the stickiness of the ECM that they deposit which increases tumour cell migration by compromising focal adhesion stability (Novo et al., 2018).

Mobilisation of certain myeloid populations, particularly neutrophils and macrophages, is key to carcinoma dissemination and metastasis and this, in part, is owing to the immunosuppressive microenvironments that these cells engender. The historical paradigm of the 'immune privilege' of the CNS is now thought to be obsolete and it is clear that immunosuppressive myeloid populations, particularly tumour-associated macrophages, drive glioma progression and therapy-resistance. Indeed, interaction of glioblastoma with surrounding astrocytes to induce an immunosuppressive environment has been linked to tumour progression (Barbero, Bajetto et al., 2002, Wang, Hong et al., 2012). More recently, it has been shown that HA can re-educate tumour-associated macrophages to a tumoursupporting phenotype (Liu, Tolg et al., 2019, Zhang, Guo et al., 2016), and this may support GBM infiltration. Supporting this line of argument, co-culture experiments of glioblastoma cells and peripheral blood cells showed that glioblastoma cells protect themselves by secreting a halo of glycosaminoglycans with HA being the main component (Gately, Muul et al., 1984). Therefore, increased HA expression by surrounding astrocytes might serve as an additional barrier to invading immune cells while simultaneously promoting glioblastoma invasion.

As well as directly promoting tumour cell migration and generating an immunosuppressive microenvironment, HA may influence glioma infiltration and therapy-resistance by promoting tumour cell stemness. HA in the nervous system has its peak during brain development with 
a subsequent decline to adulthood (Margolis, Margolis et al., 1975). Thus, HA is only abundant in the subventricular zone and rostral migratory stream of adult mice, which are thought to constitute neural stem cell niches (Lindwall, Olsson et al., 2013). Therefore, by increasing astrocyte HA secretion, mp53-expressing GBM may expand the number of stem cell niches in the adult brain, thus supporting a less proliferative stem-like phenotype capable of evading current standard treatments such as radiotherapy and chemotherapy while simultaneously increasing their capacity to invade the brain to evade surgical resection.

In conclusion, this study offers a potential mechanistic explanation for the particularly poor prognosis of GBM which are driven by the $\mathrm{p} 53^{273 \mathrm{H}}$ mutant and highlights potential druggable targets both within the tumour (Rab35, PODXL) and in the astrocytes (DGKa) which influence the ECM microenvironment through which glioma cells invade. Moreover, our finding that increased HA deposition by astrocytes is key to glioma cell infiltration will prompt further investigation into the impact of this ECM component on the immune microenvironment in the brain and the regulation of tumour stem cell maintenance and resistance to therapy. 


\section{METHODS}

\section{Cell lines, antibodies, expression vectors and reagents}

Primary patient-derived stem cell enriched cell: The E2 and G7 GBM cell lines were obtained from Colin Watts (Cambridge, now Birmingham) and chosen for their characteristic growth pattern in CD1 nude mice xenografts (Koessinger et al., 2020). Cell were originally isolated from fresh tumour tissue from anonymised patients diagnosed with glioblastoma and continued expression of glioblastoma-characteristic cellular markers was compared to previous publications using these cell lines (Ahmed et al., 2015, Birch et al., 2018, Carruthers, Ahmed et al., 2015, Fael Al-Mayhani et al., 2009). GBM cells were cultured under serum-free conditions in adDMEM/F12, $1 \% \mathrm{~B} 27,0.5 \% \mathrm{~N} 2,4 \mu \mathrm{g} / \mathrm{ml}$ heparin, $10 \mathrm{ng} / \mathrm{ml}$ fibroblast growth factor 2 (bFGF), $20 \mathrm{ng} / \mathrm{ml}$ epidermal growth factor (EGF) and 1\% Lglutamine. To encourage cell adherence as a monolayer, cell culture dishes were pre-coated with Matrigel diluted 1:40 in DMEM. Cells were incubated at $37^{\circ} \mathrm{C}, 5 \% \mathrm{CO}_{2}$ and routinely passaged every 3-4 days. Low passage numbers (3-15) were used for all experiments, and cell were routinely tested for mycoplasma contamination.

Astrocytes: Primary cultured rat cortical astrocytes were obtained from Prof. Susan Barnett (University of Glasgow) (Sorensen et al. 2008). Cells were cultured in DMEM supplemented with $10 \%$ foetal bovine serum (FBS), $1 \%$ L-glutamine and $1 \%$ Penicillin/Streptomycin in tissue-culture treated plastic dishes. Passages 1-5 were used for experiments.

Antibodies and binding-proteins: antibodies recognising Nestin (ab22035), PODXL (ab150358) and SOX2 (ab92494) were from Abcam. Other antibodies were: anti-CD63 (Pelicluster M1544), anti-p53 (Santa Cruz SC6243), anti-Rab35 (Cell Signaling 96905), anti$\beta$-actin (Sigma A1978), anti-CSPG (Sigma C8035) and anti-vinculin was generated inhouse. Bioinylated HABP (b-HABP; versican G1 domain, VG1) was from Sigma (385911).

\section{CRISPR and transfections}

For CRISPR/cas9-mediated gene knockouts, the following guide sequences were cloned into the lentiCRISPR vector (Addgene plasmid \#52961 - deposited by the Zhang lab (Shalem, Sanjana et al., 2014)):

TP53 \#1 forward CACCGCGCTATCTGAGCAGCGCTCA

TP53 \#1 reverse AAACTGAGCGCTGCTCAGATAGCGC

TP53 \#2 forward CACCGCCCCGGACGATATTGAACAA

TP53 \#2 reverse AAACTTGTTCAATATCGTCCGGGGC 
PODXL \#1 forward CACCGCAGCTCGTCCTGAACCTCAC

PODXL \#1 reverse AAACGTGAGGTTCAGGACGAGCTGC

PODXL \#2 forward CACCGGGTGTTCTCAATGCCGTTGC

PODXL \#2 reverse AAACGCAACGGCATTGAGAACACCC

Rab35 \#1 forward CACCGCTTGAAATCCACTCCGATCG

Rab35 \#1 reverse AAACCGATCGGAGTGGATTTCAAGC

Rab35 \#2 forward CACCGGAAGATGCCTACAAATTCGC

Rab35 \#2 reverse AAACGCGAATTTGTAGGCATCTTCC

Active lentiviruses were produced using HEK293FT cells as the packing line. E2 GBM cells were plated onto Matrigel, transduced with lentiviruses and selected using puromyucin (1 $\mu \mathrm{g} / \mathrm{ml})$ or blasticidin $(5 \mu \mathrm{g} / \mathrm{ml})$.

For overexpression of PODXL, the sequence for human PODXL (hPODXL) was cloned into the PQCXIZ-eGFP-C1 retroviral vector (a gift from David Bryant) and Phoenix-Ampho cells were used as the host packaging line. E2 GBM cells were plated onto Matrigel, transduced with retroviruses and selected using Zeocin $(1 \mathrm{mg} / \mathrm{ml})$.

\section{EV purification and nanoparticle tracking analysis}

GBM cells were plated into Matrigel-coated $15 \mathrm{~cm}$ tissue culture dishes at $1.5 \times 10^{6} \mathrm{cells} / \mathrm{dish}$. After $48 \mathrm{hr}$, cells were washed with PBS and $15 \mathrm{ml}$ of EV-free DMEM added. EV free media were obtained by overnight centrifugation at 100,000 g. After 48 hours, EVs were collected via differential centrifugation of cell-conditioned medium as described previously (Novo et al., 2018, Rabas, Palmer et al., 2021). The volume of $0.02 \mu \mathrm{m}$-filtered PBS used to re-suspend final EV pellets depended on the original volume of conditioned media as follows: for instance, $22 \mu \mathrm{l}$ of filtered PBS was used per $15 \mathrm{~cm}$ dish from which the EVs were collected. All EV isolations were stored at $4^{\circ} \mathrm{C}$ and used within 48 hours. Nanoparticle tracking analysis was carried out using the NanoSight LM10 instrument (Malvern Panalytical) according to the manufacturer's instructions. Re-suspended EVs were diluted 1:50 in filtered PBS before being introduced into the instrument for measurement. 
bioRxiv preprint doi: https://doi.org/10.1101/2022.02.11.480036; this version posted February 11, 2022. The copyright holder for this preprint (which was not certified by peer review) is the author/funder. All rights reserved. No reuse allowed without permission.

Glioma EVs foster a pro-invasive microenvironment

Koessinger, Novo et al.

\section{ECM generation}

Primary rat astrocytes were grown to $80 \%$ confluence in $15 \mathrm{~cm}$ dishes before being incubated in the presence or absence of EVs at a concentration of $1 \times 10^{9}$ particles $/ \mathrm{ml}$ for 72 hr. EV-treated astrocytes were re-plated at $1 \times 10^{6}$ cells/well into 6-well dishes pre-coated with $0.2 \%$ gelatin, which was subsequently crosslinked with $1 \%$ glutaraldehyde for 30 min, quenched in $1 \mathrm{M}$ glycine for $20 \mathrm{~min}$. Astrocytes were allowed to deposit ECM for 6 days in medium supplemented with $50 \mu \mathrm{g} / \mathrm{mL}$ ascorbic acid in the presence or absence of the DGK-

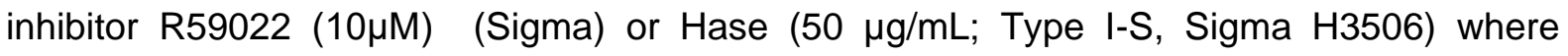
indicated. ECM was de-cellularised by incubation with PBS containing $20 \mathrm{mM} \mathrm{NH}_{4} \mathrm{OH}$ and $0.5 \%$ Triton $\mathrm{X}-100$ and stored at $4^{\circ} \mathrm{C}$ or used immediately for experiments.

\section{Immunofluorescence detection of HA and CSPG in astrocyte-deposited ECM}

ECM was generated by culturing untreated or EV pre-treated astrocytes for 7 days on gelatin-coated plates in the presence of ascorbic acid $(50 \mu \mathrm{g} / \mathrm{mL})$. Astrocyte cultures were then fixed in 4\% PFA and blocked in 1\% BSA in the absence of detergent. For labelling extracellular hyaluronic acid, samples were incubated with biotin-conjugated HABP protein (Sigma 385911), followed by streptavidin conjugated to AlexaFluor-488 (ThermoFisher S11223). Cell membranes and nuclei were counterstained using HCS CellMask Deep Red Stain (ThermoFisher H32721) and 4',6-diamidino-2-phenylindole (DAPI) respectively. For labelling extracellular chondroitin sulphate, a mouse monoclonal antibody against CSPG (Sigma C8035) was used followed by an anti-mouse secondary antibody conjugated to AlexaFluor-488. Astrocytes and the ECM they had deposited were then visualised by confocal microscopy using an Olympus Fluoview FV1000 microscope. Z-stacks were acquired from the substrate to the upper surface of the cultures at intervals of $0.5 \mu \mathrm{m}$. The channels corresponding to HABP or CSPG were thresholded and their total intensity was determined in each field-of-view. For the quantification of HABP cables, background fluorescence was subtracted and the total HABP intensity in the focal plane at the upper surface of the cells was compared between experimental arms. Values were normalised to cell number using DAPI stain.

\section{GBM cell migration on astrocyte-deposited ECM}

GBM cells were seeded onto de-cellularised ECMs at a density of $8 \times 10^{4}$ cells per well. After allowing the cells to adhere for $4 \mathrm{~h}$, the plate was transferred in a $5 \% \mathrm{CO}_{2}$ chamber at $37^{\circ} \mathrm{C}$. Images were taken every $10 \mathrm{~min}$ for $16 \mathrm{~h}$ using a Nikon time-lapse Z6011, CoolSNAP HQ camera (photometrics) and Metamorph software (molecular devices). Manual cell tracking was performed using Fiji imageJ (Schindelin, Arganda-Carreras et al., 2012). Migration 
bioRxiv preprint doi: https://doi.org/10.1101/2022.02.11.480036; this version posted February 11, 2022. The copyright holder for this preprint (which was not certified by peer review) is the author/funder. All rights reserved. No reuse allowed without permission.

Glioma EVs foster a pro-invasive microenvironment

Koessinger, Novo et al.

parameters were calculated using the ImageJ Chemotaxis and Migration tool version 1.01 (Ibidi).

\section{GBM cell migration and morphology on brain slices}

5-8 week old female C57BI/6 mice were humanely sacrificed. Upon confirmation of death, the brains from $\mathrm{DGKa}^{+/+}$or $\mathrm{DGKa}{ }^{-/,}$5-8 week old female $\mathrm{C} 57 \mathrm{BI} / 6$ mice immediately were sliced into $1 \mathrm{~mm}$ thick slices using a mouse brain slicer (Zivic instruments) and placed in Millicell $0.4 \mu \mathrm{m}$ inserts in 6 well dishes. Brain slices were maintained at $5 \% \mathrm{CO} 2,37^{\circ} \mathrm{C}$ in $\operatorname{adDMEM} / \mathrm{F} 12,1 \% \mathrm{~B} 27,0.5 \% \mathrm{~N} 2,1 \%$ Penicillin/Streptomycin. Brain slices were incubated with $\mathrm{EV}$ in $6 \mathrm{ml}$ medium for $72 \mathrm{hr}$ before being washed twice with PBS. $1 \times 10^{5}$ GFPexpressing E2 cells were plated on brain slices and allowed to attach overnight before inversion onto Lumox $35 \mathrm{~mm}$ dishes, secured with Nuclepore Track-Etch membrane and sealed with Vetbond tissue glue. EFP-expressing cells were visualised using a Nikon A1R confocal microscope with frames being captured every $10 \mathrm{~min}$ for $16 \mathrm{hr}$. For analysis of cell shape on EV-pre-treated brain slices, images of the first time point of brain slice experiments were analysed. Three representative pictures per condition were chosen. Morphological analysis was performed using the Columbus Image Data Storage and Analysis System (PerkinElmer). Five images for each condition were imported into the software and cells were identified using an automated segmentation protocol. An unbiased automated morphological analysis was then performed on a cell-by-cell basis to generate individual cell data. The width to length ratio of each cell was calculated to generate a readout for cell roundness. Width and length measurements were taken at the longest and widest points of the cell. A decrease in this ratio indicates a decrease in cell roundness.

\section{Orthotopic xenografts}

Xenograft experiments were carried out in accordance with the Animals Act 1986 (Scientific Procedures on living animals) and the regulatory guidelines of the EU Directive 2010 under project licence PPL P4A277133 and ethical review (University of Glasgow). Mice were anesthetised using isoflurane and placed in a stereotactic frame. To prevent eye desiccation, Lacrilube eye cream was applied. For analgesia, diluted Veterigesic was injected subcutaneously at a final dose of $20 \mu \mathrm{g} / \mathrm{kg}$. The skin of the surgical area was disinfected using Hydrex skin disinfectant. The mouse was then covered using sterile drapes. Prior to incision, anaesthesia depth was assessed via pedal response. Subsequently the skin was incised along the sagittal suture and periosteum was removed using sterile cotton swabs. A burr hole craniotomy was performed using an electric hand drill $3 \mathrm{~mm}$ rostral and $2 \mathrm{~mm}$ lateral of the bregma over the right hemisphere. Subsequently, GBM cells were injected (2 
$\mu \mathrm{l} / \mathrm{min}$ ) using a Hamilton syringe inserted $3 \mathrm{~mm}$ into the brain. Skin was adapted and Vetbond tissue glue applied. Mice were put in pre-warmed recovery cages and continuously monitored until mobile. For postoperative analgesia, mice were provided with Rimadylcontaining drinking water for $48 \mathrm{hr}$ postoperatively. Mice were continuously monitored for throughout the course of the experiment, and humanely sacrificed either upon display of neurological (such as hemiparesis or paraplegia) or general (hunched posture, reduced mobility, and/or weight loss $>20 \%$ ) symptoms, or timed end-point of 9 weeks following engraftment of GBM cells.

Formalin fixed, $4 \mu \mathrm{m}$ thick coronal mouse brain sections were stained for Ki67 as previously described (Koessinger et al., 2020). Slides were scanned using Leica SlidePath and engraftment analysis was conducted counting all Ki67-positive cells across coronal brain sections excluding the subventricular zone using Halo imaging software (Indica labs) with an optimised algorithm. For automated scoring of invasion to the contralateral hemisphere using Halo imaging software, slides were inspected for comparability of hemispheres- 4-5 slides per mouse from levels $50 \mu \mathrm{m}$ apart were analysed for each mouse. Both the injected (right) and contralateral (left) hemisphere were separately annotated and prevalence of Ki67 determined using the same algorithm as for the engraftment analysis for each hemisphere, respectively. 


\section{ACKNOWLEDGEMENTS}

This work was funded by Cancer Research UK and Breast Cancer Now. We acknowledge the Cancer Research UK Glasgow Centre (C596/A18076) and the BSU facilities at the Cancer Research UK Beatson Institute (C596/A17196). Many thanks to Colin Nixon and the CRUK-Beatson Institute's histology lab for the sectioning and staining of brain slices.

\section{AUTHOR CONTRIBUTIONS}

D.K., D.N., A.K., D.Z., L.M., M.M. and J.B. performed the experiments and analysed experimental data. J.N. and J.B. supervised the experimental work. A.C. contributed to the conceptual design and planning of experiments, and provision of reagents. D.K. and J.N. prepared the figures and wrote the manuscript.

\section{DECLARATION OF INTERESTS}

The authors declare no competing financial interests.

\section{DATA AVAILABILITY}

The data supporting the findings of this study are available within the article and its supplementary information files and from the corresponding author upon request. 
bioRxiv preprint doi: https://doi.org/10.1101/2022.02.11.480036; this version posted February 11, 2022. The copyright holder for this preprint (which was not certified by peer review) is the author/funder. All rights reserved. No reuse allowed without permission.

Glioma EVs foster a pro-invasive microenvironment

Koessinger, Novo et al.

\section{FIGURE LEGENDS}

Figure 1: EVs from infiltrative GMB cells foster a pro-migratory microenvironment

A, B) Coronal brain slices from $\mathrm{DGK}^{+/+}$or $\mathrm{DGK}^{-/-}$mice were treated for $72 \mathrm{hr}$ with equal quantities of EVs from highly infiltrative (E2), less infiltrative (G7) patient-derived glioma stem-like cells or were left untreated (-). GFP-expressing GBM (E2) cells were then plated onto brain slices and their migration speed $(A)$ and shape $(B)$ were determined using timelapse fluorescence microscopy and high-content imaging respectively. In (A) bars are mean \pm sem of the mean values from 3 independent experiments (indicated by the dots); 60 cells per condition were tracked in each experiment, paired t-test $(n=3)$. In (B) bars are median, boxes denote interquartile range and whiskers the $10-90 \%$ range. Unpaired t-test. Bars are $50 \mu \mathrm{m}$.

C) Astrocytes isolated from rat brain cortex were treated for $72 \mathrm{hr}$ with EVs from E2 or G7 GBM cells or were left untreated (-). Astrocytes were then allowed to deposit ECM for 6 days in the presence of $10 \mu \mathrm{M}$ R59022 (DGKa inhibitor) or vehicle control (DMSO). ECM was then decellularized and E2 (left graph and micrographs) or G7 (right graph) plated onto these. The migration speed of these GBM cells on astrocyte-derived ECM was then determined using time-lapse microscopy. Bars are mean \pm sem of the mean values from 3 independent experiments (indicated by the dots); 60 cells per condition were tracked in each experiment, paired t-test $(n=3)$; ns, not significant.

\section{Figure 2: Deletion of $\mathrm{p53}^{273 \mathrm{H}}$ abrogates the ability of GBM cells to produce EVs which encourage astrocytes to deposit pro-migratory ECM}

(A) E2 GBM cells were transfected with lentiviral CRISPR/Cas9 vectors containing 2 independent guide sequences targeting p53 (p53-ko \#1 and \#2) or empty vector control (CRISPR-ctrl). Deletion of $\mathrm{p} 53^{273 \mathrm{H}}$ and expression of stem cell markers, nestin and SOX2 were confirmed by Western blotting with $\beta$-actin and vinculin as loading controls.

(B) The growth of p53-ko \#1 and \#2 cells was compared with CRISPR-ctrl cells using Incucyte live cell imaging. Representative experiment shown. Values represent the mean of three technical repeats.

(C) The number and size-distribution of EVs from CRISPR-ctrl and p53-ko cells was analysed using nanoparticle tracking. Each line represents the mean of three independent experiments.

(D) The PODXL content of CRISPR-ctrl and p53-ko cells and EVs released from them was determined using Western blotting. $\beta$-actin and CD63 are loading controls for the cell extracts and purified EVs respectively. 
(E) 'Recipient' astrocytes (Astrocyte recip.) isolated from rat brain cortex were treated for 72 hr with EVs from CRISPR-ctrl and p53-ko cells 'donor' GBM cells. Astrocytes were then allowed to deposit ECM for 6 days in the presence of $10 \mu \mathrm{M}$ R59022 (DGKa inhibitor) or vehicle control (DMSO). The migration speed of E2 GBM cells plated onto this astrocytederived ECM was determined using time-lapse microscopy. Bars are mean \pm sem of the mean values from 3 independent experiments (indicated by the dots); 60 cells per condition were tracked in each experiment, paired t-test $(n=3)$; ns, not significant.

\section{Figure 3: Manipulation of PODXL content of GBM cell-derived EVs}

E2 GBM cells were stably transfected with lentiviral vectors encoding GFP-tagged PODXL (hPODXL-GFP) or GFP (A), or with CRISPR/Cas9 vectors containing 2 independent guide sequences targeting PODXL (PODXL-ko \#1 and \#2) (B), Rab35 (Rab35-ko\#1 and \#2) (C) or empty vector control (CRISPR-ctrl). PODXL overexpression, deletion of PODXL and Rab35, expression of stem cell markers, nestin and SOX2 were confirmed by Western blotting with $\beta$-actin and vinculin as loading controls. PODXL content of EVs was determined by Western blotting using CD63 as loading control. Cell proliferation and the number and sizedistribution of EV were determined using Incucyte imaging and nanoparticle tracking respectively as for Fig. $2 \mathrm{~B} \& \mathrm{C}$.

Figure 4: The PODXL content of EVs is critical to support deposition of pro-migratory ECM by astrocytes

(A-C) 'Recipient' astrocytes (Astro. recip.) were treated for $72 \mathrm{hr}$ with EVs from 'donor' GBM cells in which PODXL or Rab35 levels were manipulated (as described in Fig. 3). Astrocytes were then allowed to deposit ECM for 6 days in the presence of $10 \mu \mathrm{M}$ R59022 (DGKa inhibitor) or vehicle control (DMSO). The migration speed of E2 GBM cells plated onto this astrocyte-deposited ECM was determined using time-lapse microscopy.

(D) 'Recipient' brain slices (Brain recip.) from DGKa ${ }^{+/+}$mice were incubated with EVs from CRISPR-ctrl, PODXL-ko or Rab35-ko 'donor' GBM cells for $72 \mathrm{hr}$. GFP-expressing GBM (E2) cells were then plated onto brain slices and their migration speed determined using time-lapse fluorescence microscopy. In A-D, bars are mean \pm sem of the mean values from 3 independent experiments (indicated by the dots); 60 cells per condition were tracked in each experiment, paired t-test $(n=3)$; ns, not significant.

Figure 5: EVs from GBM cells promote GBM migration by influencing the HA content of astrocyte-deposited ECM 
(A, B) 'Recipient' astrocytes were incubated with EVs from G7 or E2 GBM 'donor' cells (A), or E2 GBM cells in which p53, PODXL or Rab35 had been deleted using CRISPR/Cas9 approaches (B). EV-pretreated astrocytes were then allowed to deposit ECM for 6 days. Astrocyte-deposited ECM was then stained with HABP (green) in combination with cell mask (red) and imaged using fluorescence confocal microscopy; both $\mathrm{x} / \mathrm{y}$ (upper panels) and $\mathrm{x} / \mathrm{y} / \mathrm{z}$ (lower panels) projections are displayed. Bars $50 \mu \mathrm{m}$. The intensity of HABP staining present on the upper surface of the astrocyte cultures was quantified using Image J. Bars are mean \pm sem of the mean values from 3 independent experiments (indicated by the dots); 7 fields per condition were quantified in each experiment, paired t-test $(n=3)$; ns, not significant.

(C, D) Recipient astrocytes (Astro. recip.) were treated with EVs from E2 GBM cells for 72 $\mathrm{hr}$, and then allowed to deposit ECM for 6 days. Type I-S Hase $(50 \mu \mathrm{g} / \mathrm{mL})$ was added where indicated on the $5^{\text {th }}$ day of culture. In (D) the HA content of the ECM was assessed with HABP-staining (green), followed by quantification using Image $J$ as for (A). In (C) the migration speed of E2 GBM cells plated onto Hase-treated and control (ctrl) ECM was determined using time-lapse microscopy. Bars are mean \pm sem of the mean values from 3 independent experiments (indicated by the dots); 50 cells per condition were tracked in each experiment, paired t-test $(n=3)$; ns, not significant.

Figure 6: PODXL-expression is required for long-range infiltrative migration of GBM cells

CRISPR control (CRISPR-ctrl) or PODXL knockout (PODXL-ko) GBM cells were injected into the right (ipsilateral) striatum of CD1 nude mice. 9 weeks following injection, brains were fixed and the distribution of tumour cells between the ipsilateral (Ipsi.) and contralateral (Contra.) determined using Ki67 staining followed by automated image analysis. Cells remaining in the ipsilateral hemisphere and those that have migrated to the contralateral hemisphere are highlighted with blue and red arrows respectively $(A)$. The number of Ki67positive cells present in the contralateral hemisphere is expressed as a ratio of those remaining in the ipsilateral hemisphere $(B)$. Bars are mean \pm sem. The dots represent Ki67positive cells in the contralateral hemisphere expressed as a ratio of the Ki67-positive cells in the ipsilateral hemisphere from each individual brain slice. Statistic is unpaired t-test, Welch's correction. Bar in $(A)$ is $1 \mathrm{~mm}$.

Figure 7: Schematic representation of an intercellular communication cascade through which mutant $\mathrm{p53}^{273 \mathrm{H}}$ drives GBM infiltration 
Three sequential and distinct mechanistic events contribute to a cascade through which a gain-of-function p53 mutant, p53 ${ }^{273 \mathrm{H}}$ may drive infiltrative behaviour in GBM: [1] Expression of $\mathrm{p} 53^{273 \mathrm{H}}$ acts in combination with Rab35 to control sorting of PODXL into EVs; [2] Astrocytes respond to PODXL-containing EVs by depositing ECM with increased HA content. This response is dependent on DGKa; [3] GBM cells respond to HA-rich ECM by increasing their invasive behaviour and this is associated with long-distance infiltration of the brain. 
bioRxiv preprint doi: https://doi.org/10.1101/2022.02.11.480036; this version posted February 11, 2022. The copyright holder for this preprint (which was not certified by peer review) is the author/funder. All rights reserved. No reuse allowed without permission.

Glioma EVs foster a pro-invasive microenvironment Koessinger, Novo et al. 
bioRxiv preprint doi: https://doi.org/10.1101/2022.02.11.480036; this version posted February 11, 2022. The copyright holder for this preprint (which was not certified by peer review) is the author/funder. All rights reserved. No reuse allowed without permission.

Glioma EVs foster a pro-invasive microenvironment

Koessinger, Novo et al.

\section{REFERENCES}

Abatangelo G, Vindigni V, Avruscio G, Pandis L, Brun P (2020) Hyaluronic Acid: Redefining Its Role. Cells 9

Ahmed SU, Carruthers R, Gilmour L, Yildirim S, Watts C, Chalmers AJ (2015) Selective Inhibition of Parallel DNA Damage Response Pathways Optimizes Radiosensitization of Glioblastoma Stem-like Cells. Cancer Res 75: 4416-28

Ahn JH, Kim TJ, Lee JH, Choi JH (2017) Mutant p53 stimulates cell invasion through an interaction with Rad21 in human ovarian cancer cells. Sci Rep 7: 9076

Barbero S, Bajetto A, Bonavia R, Porcile C, Piccioli P, Pirani P, Ravetti JL, Zona G, Spaziante R, Florio T, Schettini G (2002) Expression of the chemokine receptor CXCR4 and its ligand stromal cell-derived factor 1 in human brain tumors and their involvement in glial proliferation in vitro. Ann N Y Acad Sci 973: $60-9$

Birch JL, Strathdee K, Gilmour L, Vallatos A, McDonald L, Kouzeli A, Vasan R, Qaisi AH, Croft DR, Crighton D, Gill K, Gray CH, Konczal J, Mezna M, McArthur D, Schuttelkopf AW, McConnell P, Sime M, Holmes WM, Bower J et al. (2018) A Novel Small-Molecule Inhibitor of MRCK Prevents RadiationDriven Invasion in Glioblastoma. Cancer Res 78: 6509-6522

Broekman ML, Maas SLN, Abels ER, Mempel TR, Krichevsky AM, Breakefield XO (2018) Multidimensional communication in the microenvirons of glioblastoma. Nat Rev Neurol 14: 482-495 Carruthers R, Ahmed SU, Strathdee K, Gomez-Roman N, Amoah-Buahin E, Watts C, Chalmers AJ (2015) Abrogation of radioresistance in glioblastoma stem-like cells by inhibition of ATM kinase. Mol Oncol 9: 192-203

Ceccarelli M, Barthel FP, Malta TM, Sabedot TS, Salama SR, Murray BA, Morozova O, Newton Y, Radenbaugh A, Pagnotta SM, Anjum S, Wang J, Manyam G, Zoppoli P, Ling S, Rao AA, Grifford M, Cherniack AD, Zhang H, Poisson L et al. (2016) Molecular Profiling Reveals Biologically Discrete Subsets and Pathways of Progression in Diffuse Glioma. Cell 164: 550-63

Costa-Silva B, Aiello NM, Ocean AJ, Singh S, Zhang H, Thakur BK, Becker A, Hoshino A, Mark MT, Molina H, Xiang J, Zhang T, Theilen TM, Garcia-Santos G, Williams C, Ararso Y, Huang Y, Rodrigues G, Shen TL, Labori KJ et al. (2015) Pancreatic cancer exosomes initiate pre-metastatic niche formation in the liver. Nat Cell Biol 17: 816-26

de Chaffoy de Courcelles DC, Roevens P, Van Belle H (1985) R 59 022, a diacylglycerol kinase inhibitor. Its effect on diacylglycerol and thrombin-induced $\mathrm{C}$ kinase activation in the intact platelet. $J$ Biol Chem 260: 15762-70

de Vrij J, Maas SL, Kwappenberg KM, Schnoor R, Kleijn A, Dekker L, Luider TM, de Witte LD, Litjens M, van Strien ME, Hol EM, Kroonen J, Robe PA, Lamfers ML, Schilham MW, Broekman ML (2015) Glioblastoma-derived extracellular vesicles modify the phenotype of monocytic cells. Int J Cancer 137: $1630-42$

Deen AJ, Rilla K, Oikari S, Karna R, Bart G, Hayrinen J, Bathina AR, Ropponen A, Makkonen K, Tammi RH, Tammi MI (2014) Rab10-mediated endocytosis of the hyaluronan synthase HAS3 regulates hyaluronan synthesis and cell adhesion to collagen. J Biol Chem 289: 8375-89

Domenis R, Cesselli D, Toffoletto B, Bourkoula E, Caponnetto F, Manini I, Beltrami AP, lus T, Skrap M, Di Loreto C, Gri G (2017) Systemic T Cells Immunosuppression of Glioma Stem Cell-Derived Exosomes Is Mediated by Monocytic Myeloid-Derived Suppressor Cells. PLoS One 12: e0169932

Drumm MR, Dixit KS, Grimm S, Kumthekar P, Lukas RV, Raizer JJ, Stupp R, Chheda MG, Kam KL, McCord M, Sachdev S, Kruser T, Steffens A, Javier R, McCortney K, Horbinski C (2020) Extensive brainstem infiltration, not mass effect, is a common feature of end-stage cerebral glioblastomas. Neuro Oncol 22: 470-479

Fael Al-Mayhani TM, Ball SL, Zhao JW, Fawcett J, Ichimura K, Collins PV, Watts C (2009) An efficient method for derivation and propagation of glioblastoma cell lines that conserves the molecular profile of their original tumours. J Neurosci Methods 176: 192-9

Gabrusiewicz K, Li X, Wei J, Hashimoto Y, Marisetty AL, Ott M, Wang F, Hawke D, Yu J, Healy LM, Hossain A, Akers JC, Maiti SN, Yamashita S, Shimizu Y, Dunner K, Zal MA, Burks JK, Gumin J, Nwajei F 
bioRxiv preprint doi: https://doi.org/10.1101/2022.02.11.480036; this version posted February 11, 2022. The copyright holder for this preprint (which was not certified by peer review) is the author/funder. All rights reserved. No reuse allowed without permission.

Glioma EVs foster a pro-invasive microenvironment

Koessinger, Novo et al.

et al. (2018) Glioblastoma stem cell-derived exosomes induce M2 macrophages and PD-L1 expression on human monocytes. Oncoimmunology 7: e1412909

Gately CL, Muul LM, Greenwood MA, Papazoglou S, Dick SJ, Kornblith PL, Smith BH, Gately MK (1984) In vitro studies on the cell-mediated immune response to human brain tumors. II. Leukocyteinduced coats of glycosaminoglycan increase the resistance of glioma cells to cellular immune attack. J Immunol 133: 3387-95

Hellwinkel JE, Redzic JS, Harland TA, Gunaydin D, Anchordoquy TJ, Graner MW (2016) Gliomaderived extracellular vesicles selectively suppress immune responses. Neuro Oncol 18: 497-506

Jothy S (2003) CD44 and its partners in metastasis. Clin Exp Metastasis 20: 195-201

Kitamura T, Qian BZ, Pollard JW (2015) Immune cell promotion of metastasis. Nat Rev Immunol 15: 73-86

Kobayashi T, Chanmee T, Itano N (2020) Hyaluronan: Metabolism and Function. Biomolecules 10 Koessinger AL, Koessinger D, Stevenson K, Cloix C, Mitchell L, Nixon C, Gomez-Roman N, Chalmers AJ, Norman JC, Tait SWG (2020) Quantitative in vivo bioluminescence imaging of orthotopic patientderived glioblastoma xenografts. Sci Rep 10: 15361

Kovalenko A, Sanin A, Kosmas K, Zhang L, Wang J, AkI EW, Giannikou K, Probst CK, Hougard TR, Rue RW, Krymskaya VP, Asara JM, Lam HC, Kwiatkowski DJ, Henske EP, Filippakis H (2021) Therapeutic Targeting of DGKA-Mediated Macropinocytosis Leads to Phospholipid Reprogramming in Tuberous Sclerosis Complex. Cancer Res 81: 2086-2100

Lindwall C, Olsson M, Osman AM, Kuhn HG, Curtis MA (2013) Selective expression of hyaluronan and receptor for hyaluronan mediated motility (Rhamm) in the adult mouse subventricular zone and rostral migratory stream and in ischemic cortex. Brain Res 1503: 62-77

Liu M, Tolg C, Turley E (2019) Dissecting the Dual Nature of Hyaluronan in the Tumor Microenvironment. Front Immunol 10: 947

Manini I, Caponnetto F, Bartolini A, lus T, Mariuzzi L, Di Loreto C, Beltrami AP, Cesselli D (2018) Role of Microenvironment in Glioma Invasion: What We Learned from In Vitro Models. Int J Mol Sci 19

Margolis RU, Margolis RK, Chang LB, Preti C (1975) Glycosaminoglycans of brain during development. Biochemistry 14: 85-8

Miller CR, Perry A (2007) Glioblastoma. Arch Pathol Lab Med 131: 397-406

Muller PA, Caswell PT, Doyle B, Iwanicki MP, Tan EH, Karim S, Lukashchuk N, Gillespie DA, Ludwig RL, Gosselin P, Cromer A, Brugge JS, Sansom OJ, Norman JC, Vousden KH (2009) Mutant p53 drives invasion by promoting integrin recycling. Cell 139: 1327-41

Novo D, Heath N, Mitchell L, Caligiuri G, MacFarlane A, Reijmer D, Charlton L, Knight J, Calka M, McGhee E, Dornier E, Sumpton D, Mason S, Echard A, Klinkert K, Secklehner J, Kruiswijk F, Vousden $\mathrm{K}$, Macpherson IR, Blyth K et al. (2018) Mutant p53s generate pro-invasive niches by influencing exosome podocalyxin levels. Nat Commun 9: 5069

Olive KP, Tuveson DA, Ruhe ZC, Yin B, Willis NA, Bronson RT, Crowley D, Jacks T (2004) Mutant p53 gain of function in two mouse models of Li-Fraumeni syndrome. Cell 119: 847-60

Osswald M, Jung E, Sahm F, Solecki G, Venkataramani V, Blaes J, Weil S, Horstmann H, Wiestler B, Syed $M$, Huang L, Ratliff $M$, Karimian Jazi K, Kurz FT, Schmenger T, Lemke D, Gommel M, Pauli $M$, Liao Y, Haring $P$ et al. (2015) Brain tumour cells interconnect to a functional and resistant network. Nature 528: 93-8

Padua D, Massague J (2009) Roles of TGFbeta in metastasis. Cell Res 19: 89-102

Park JB, Kwak HJ, Lee SH (2008) Role of hyaluronan in glioma invasion. Cell Adh Migr 2: 202-7

Pibuel MA, Poodts D, Diaz M, Hajos SE, Lompardia SL (2021) The scrambled story between hyaluronan and glioblastoma. J Biol Chem 296: 100549

Pinto G, Saenz-de-Santa-Maria I, Chastagner P, Perthame E, Delmas C, Toulas C, Moyal-JonathanCohen E, Brou C, Zurzolo C (2021) Patient-derived glioblastoma stem cells transfer mitochondria through tunneling nanotubes in tumor organoids. Biochem J 478: 21-39

Powell E, Piwnica-Worms D, Piwnica-Worms H (2014) Contribution of p53 to metastasis. Cancer Discov 4: 405-14 
bioRxiv preprint doi: https://doi.org/10.1101/2022.02.11.480036; this version posted February 11, 2022. The copyright holder for this preprint (which was not certified by peer review) is the author/funder. All rights reserved. No reuse allowed without permission.

Glioma EVs foster a pro-invasive microenvironment

Koessinger, Novo et al.

Rabas N, Palmer S, Mitchell L, Ismail S, Gohlke A, Riley JS, Tait SWG, Gammage P, Soares LL, Macpherson IR, Norman JC (2021) PINK1 drives production of mtDNA-containing extracellular vesicles to promote invasiveness. $J$ Cell Biol 220

Rainero E, Caswell PT, Muller PA, Grindlay J, McCaffrey MW, Zhang Q, Wakelam MJ, Vousden KH, Graziani A, Norman JC (2012) Diacylglycerol kinase alpha controls RCP-dependent integrin trafficking to promote invasive migration. J Cell Biol 196: 277-95

Ruoslahti E (1996) Brain extracellular matrix. Glycobiology 6: 489-92

Schindelin J, Arganda-Carreras I, Frise E, Kaynig V, Longair M, Pietzsch T, Preibisch S, Rueden C, Saalfeld S, Schmid B, Tinevez JY, White DJ, Hartenstein V, Eliceiri K, Tomancak P, Cardona A (2012) Fiji: an open-source platform for biological-image analysis. Nat Methods 9: 676-82

Schulz-Heddergott R, Stark N, Edmunds SJ, Li J, Conradi LC, Bohnenberger H, Ceteci F, Greten FR, Dobbelstein M, Moll UM (2018) Therapeutic Ablation of Gain-of-Function Mutant p53 in Colorectal Cancer Inhibits Stat3-Mediated Tumor Growth and Invasion. Cancer Cell 34: 298-314 e7

Shalem O, Sanjana NE, Hartenian E, Shi X, Scott DA, Mikkelson T, HeckI D, Ebert BL, Root DE, Doench JG, Zhang F (2014) Genome-scale CRISPR-Cas9 knockout screening in human cells. Science 343: 84-87 Treps L, Perret R, Edmond S, Ricard D, Gavard J (2017) Glioblastoma stem-like cells secrete the proangiogenic VEGF-A factor in extracellular vesicles. J Extracell Vesicles 6: 1359479

Wang SC, Hong JH, Hsueh C, Chiang CS (2012) Tumor-secreted SDF-1 promotes glioma invasiveness and TAM tropism toward hypoxia in a murine astrocytoma model. Lab Invest 92: 151-62

Weissmueller S, Manchado E, Saborowski M, Morris JPt, Wagenblast E, Davis CA, Moon SH, Pfister NT, Tschaharganeh DF, Kitzing T, Aust D, Markert EK, Wu J, Grimmond SM, Pilarsky C, Prives C, Biankin AV, Lowe SW (2014) Mutant p53 drives pancreatic cancer metastasis through cellautonomous PDGF receptor beta signaling. Cell 157: 382-394

Wortzel I, Dror S, Kenific CM, Lyden D (2019) Exosome-Mediated Metastasis: Communication from a Distance. Dev Cell 49: 347-360

Zappulli V, Friis KP, Fitzpatrick Z, Maguire CA, Breakefield XO (2016) Extracellular vesicles and intercellular communication within the nervous system. J Clin Invest 126: 1198-207

Zhang G, Guo L, Yang C, Liu Y, He Y, Du Y, Wang W, Gao F (2016) A novel role of breast cancerderived hyaluronan on inducement of $\mathrm{M} 2$-like tumor-associated macrophages formation. Oncoimmunology 5: e1172154 
A

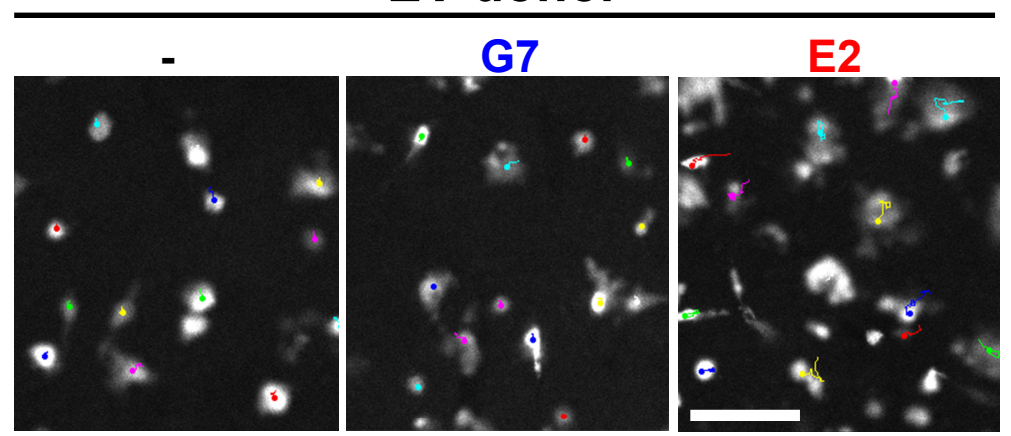

B

EV donor

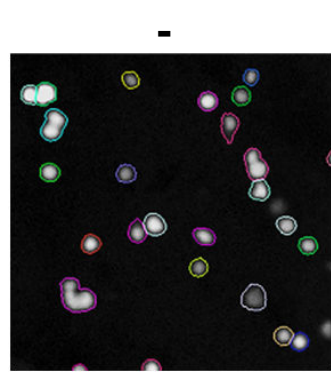

G7

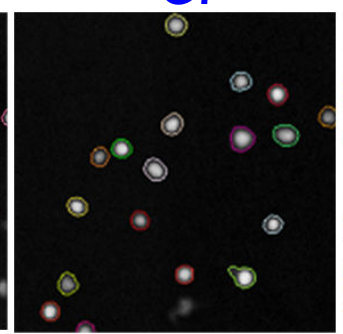

E2

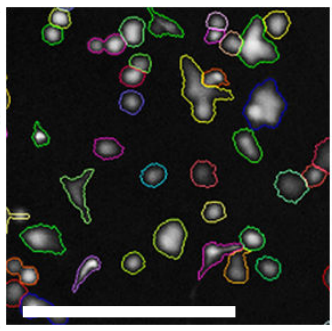

EV donor:

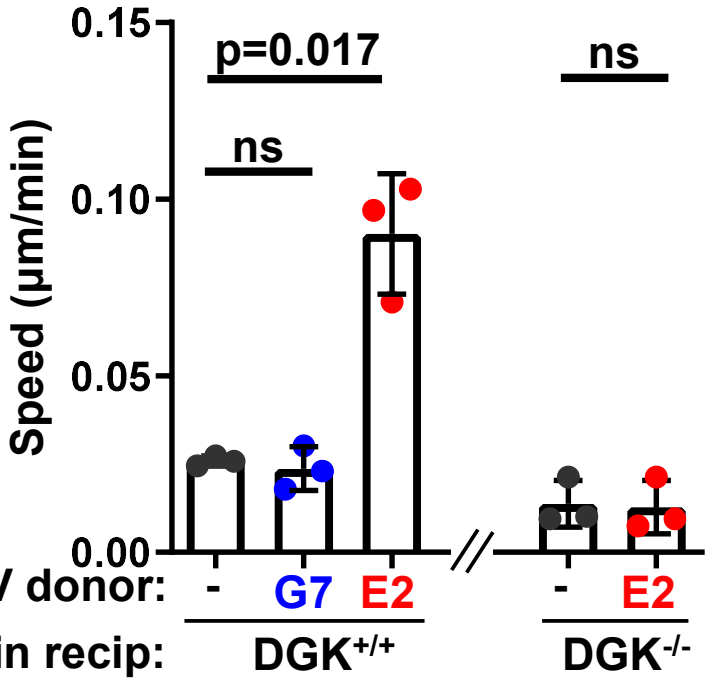

C

EV donor:
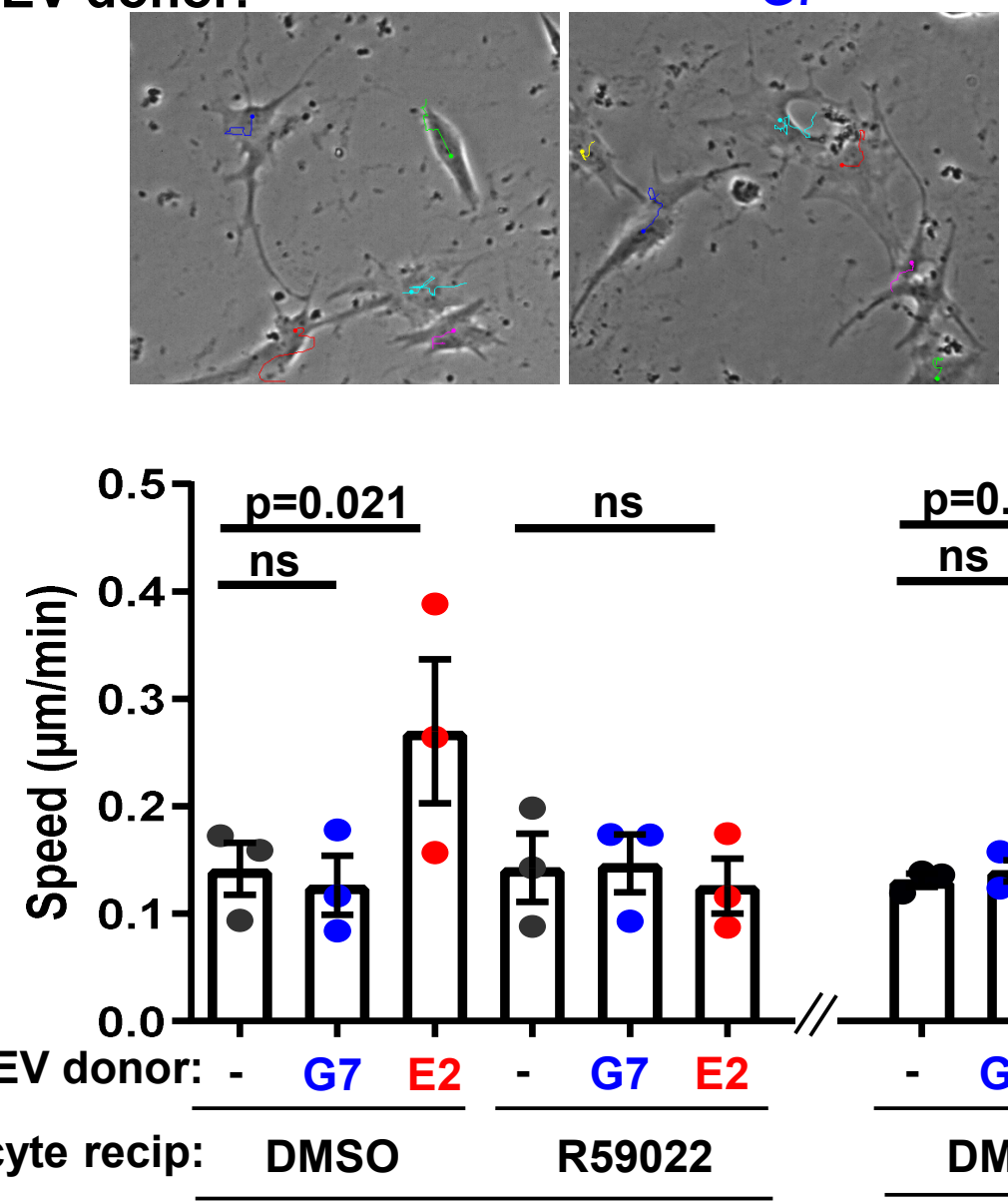

GBM migration:
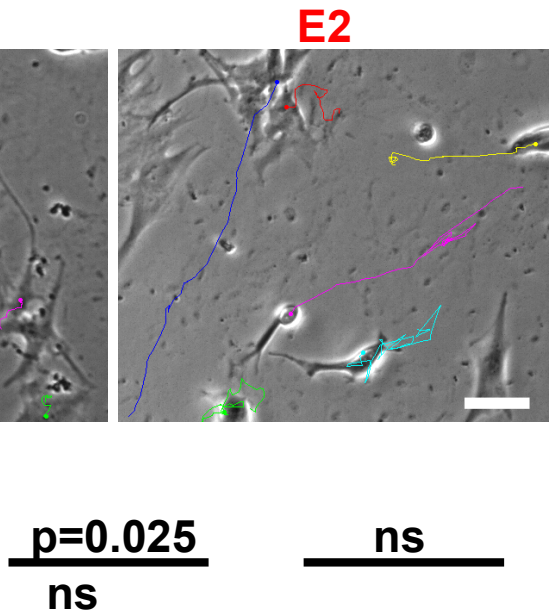

$p<0.001$

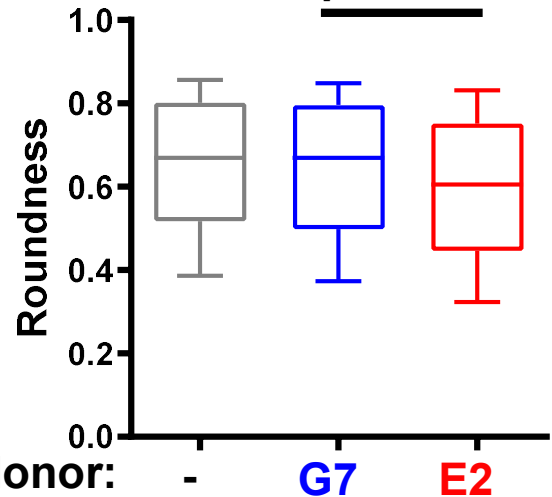

Astrocyte recip: DMSO

R59022 
A
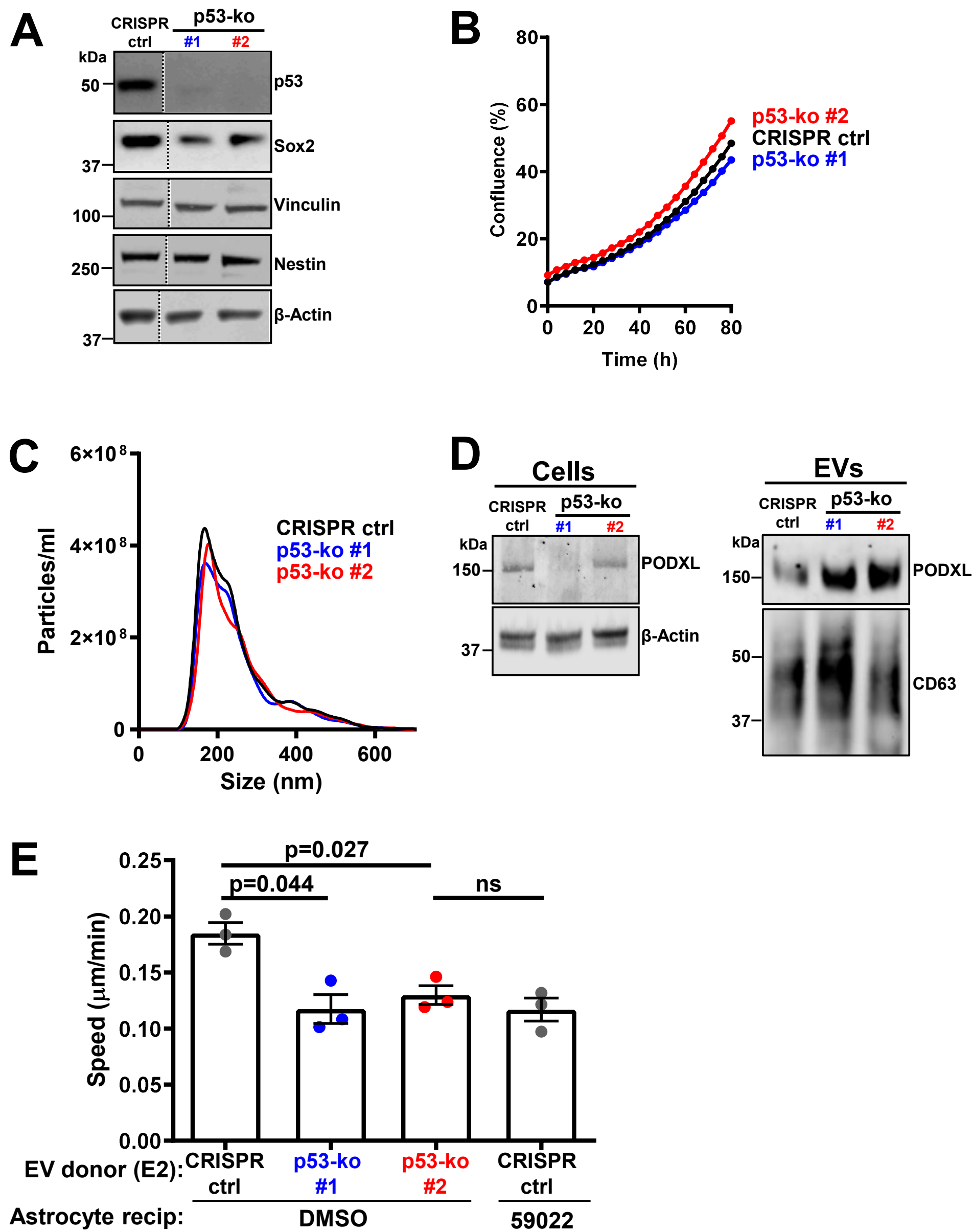
A Cells

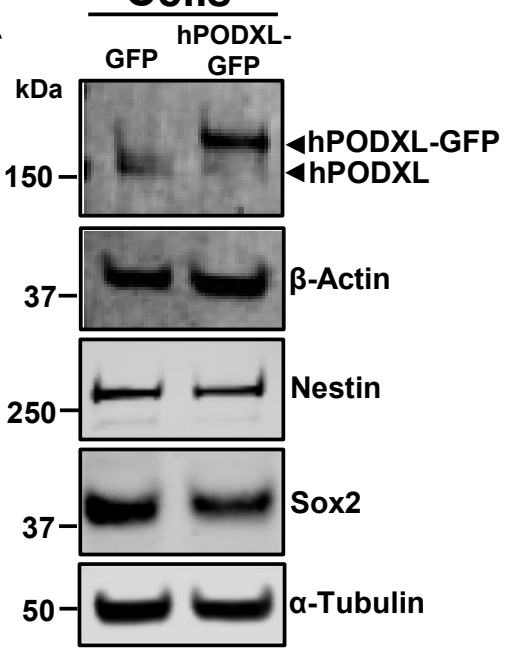

B

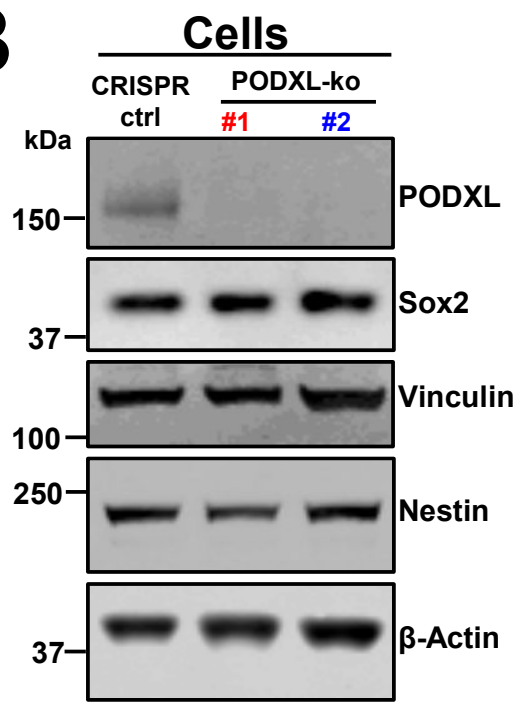

C

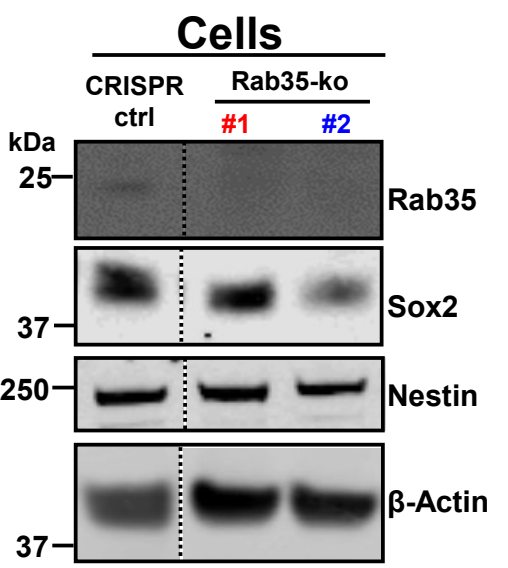

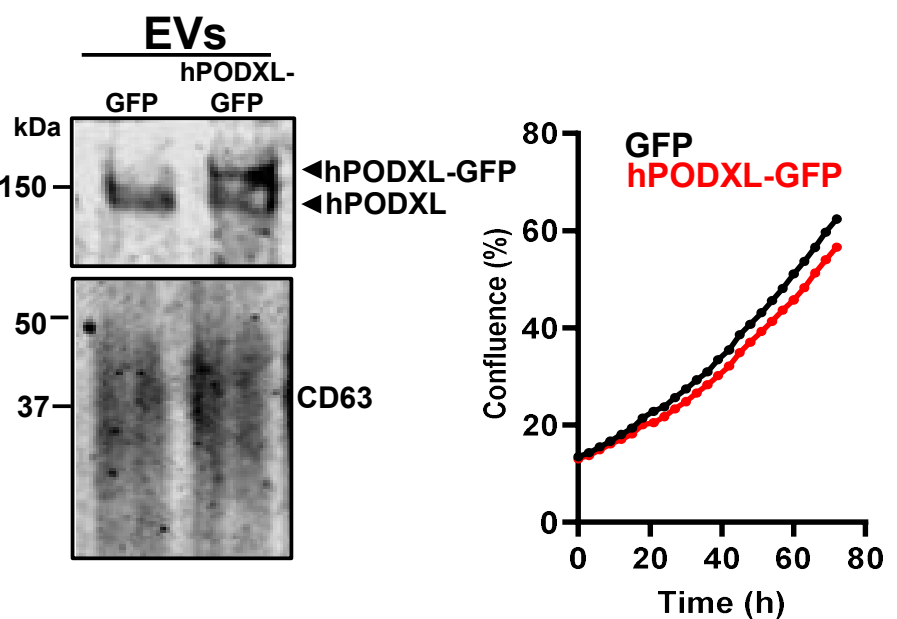
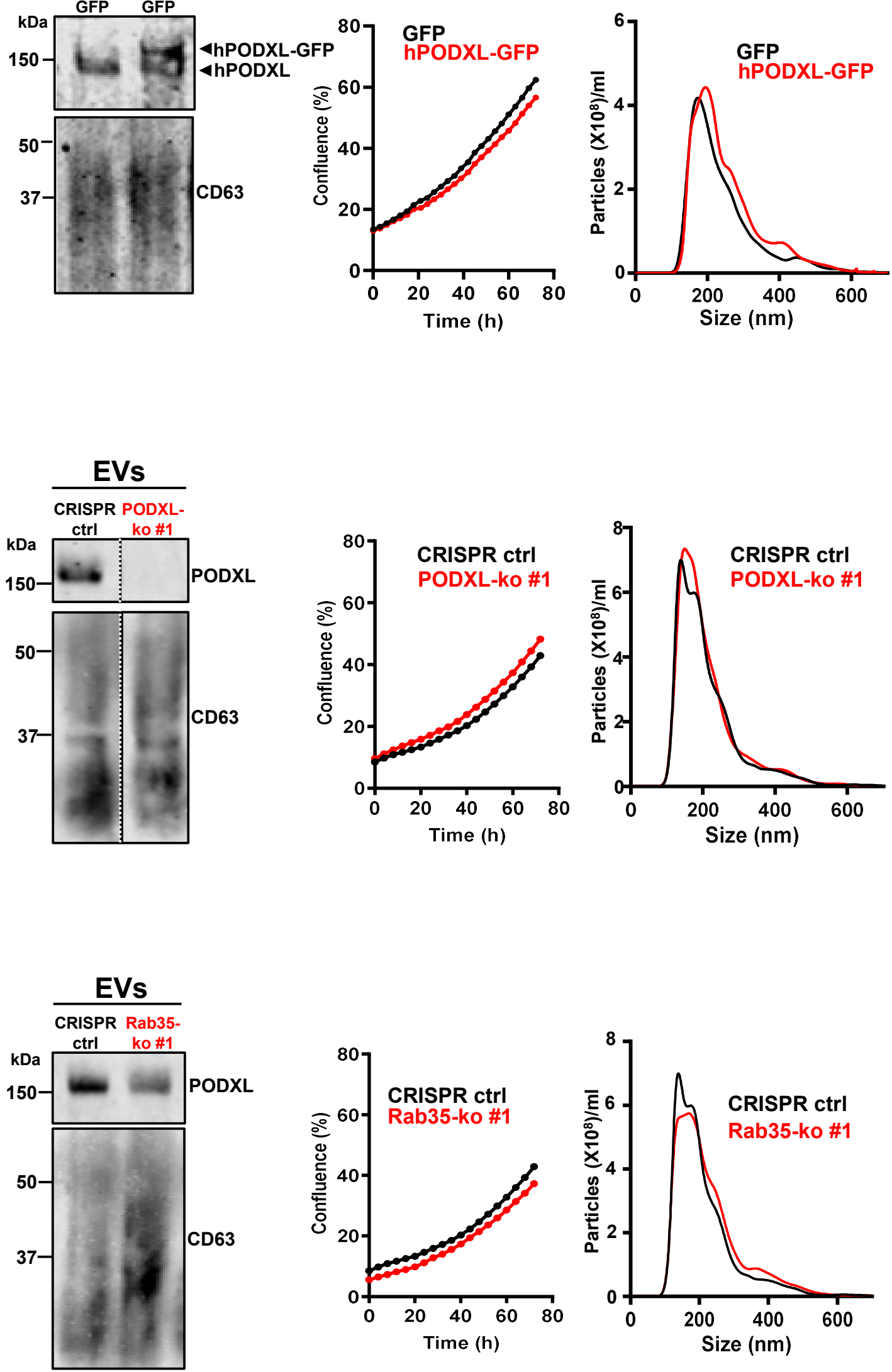

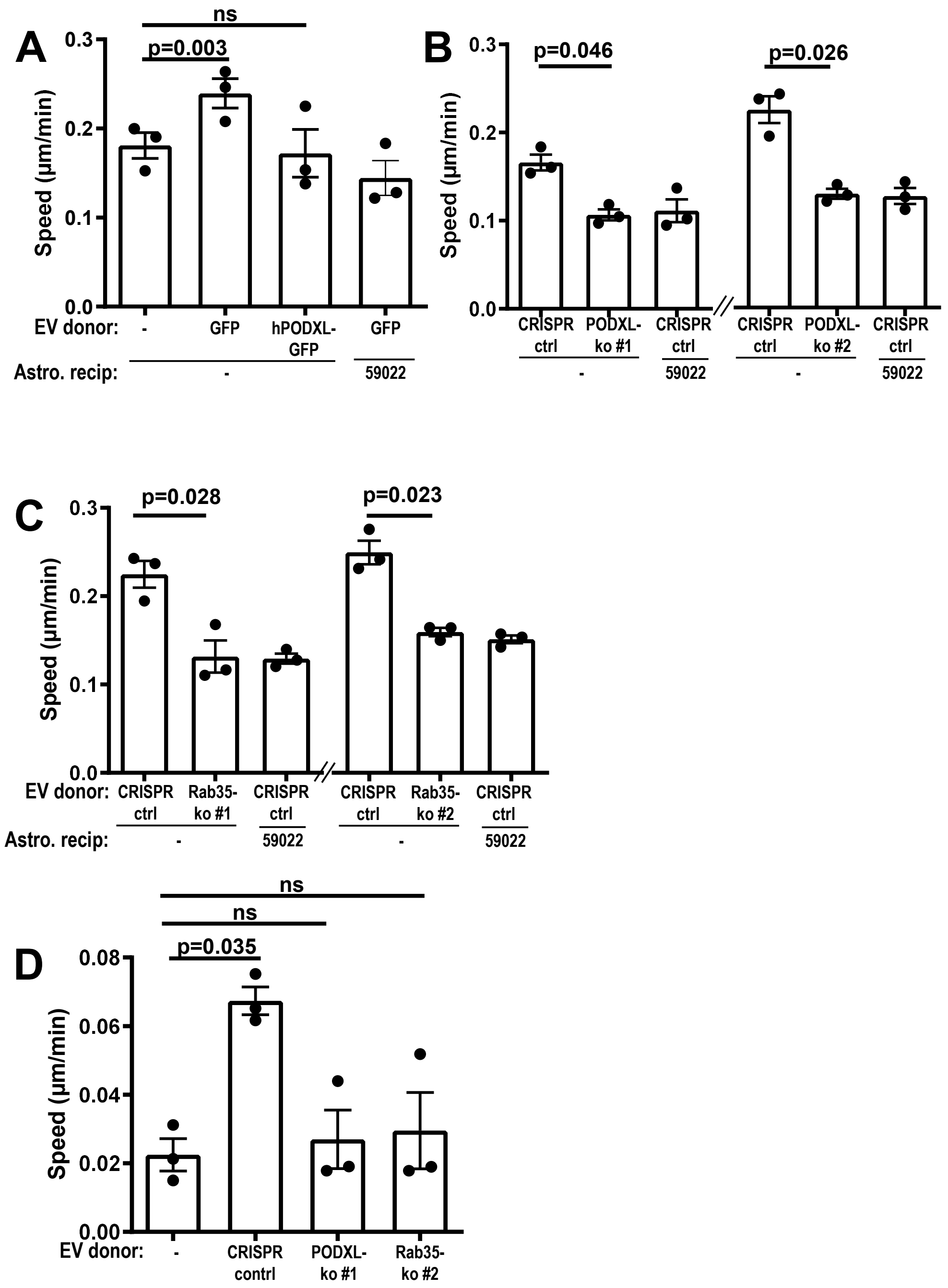

Brain recip:

DGK $\alpha^{+/+}$ 


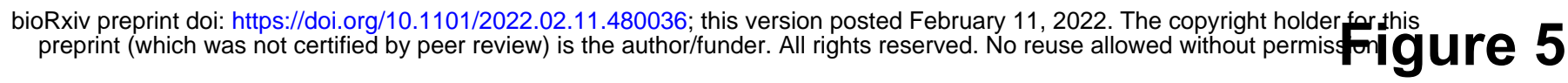

A

\section{EV donor}
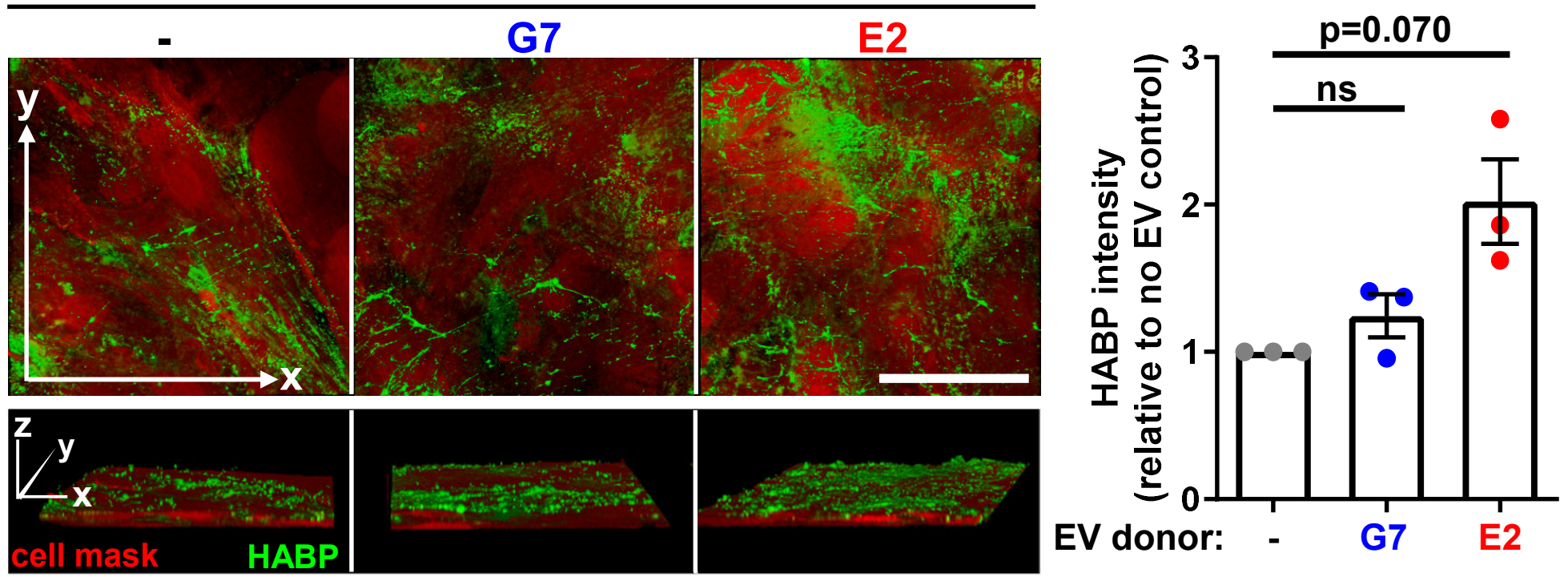

EV donor
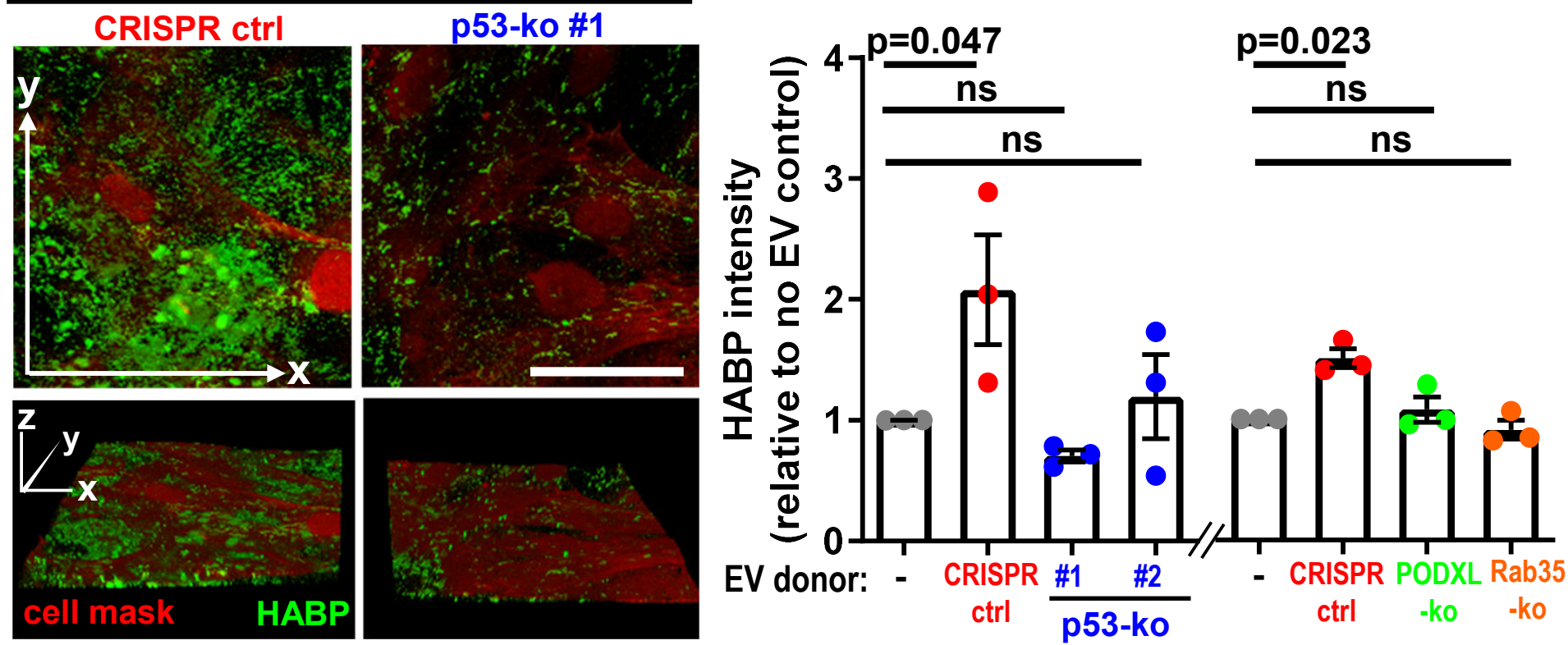

C
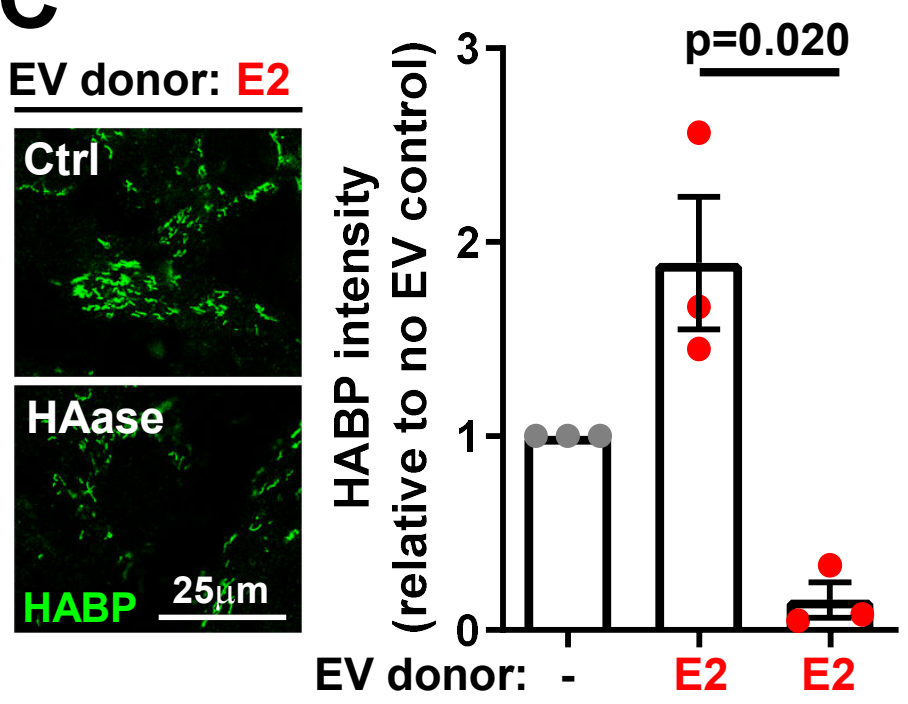

Astro. recip.: Ctrl $\overline{\text { HAase }}$

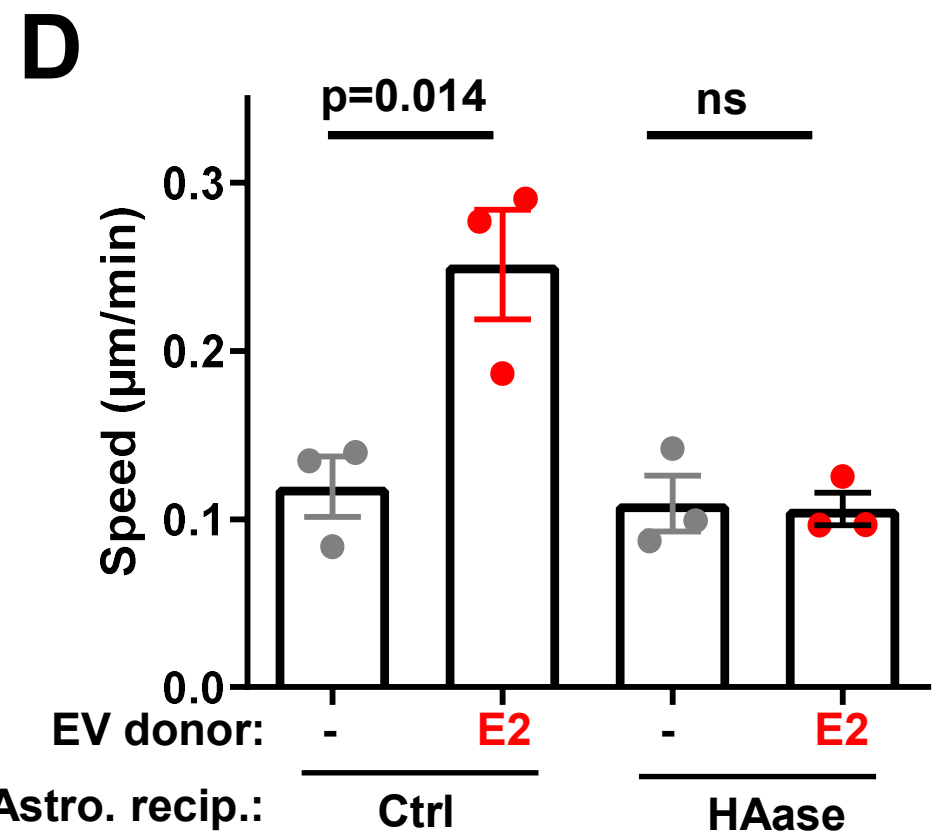


A

\section{Injected cells (E2)}

\section{CRISPR-ctrl}
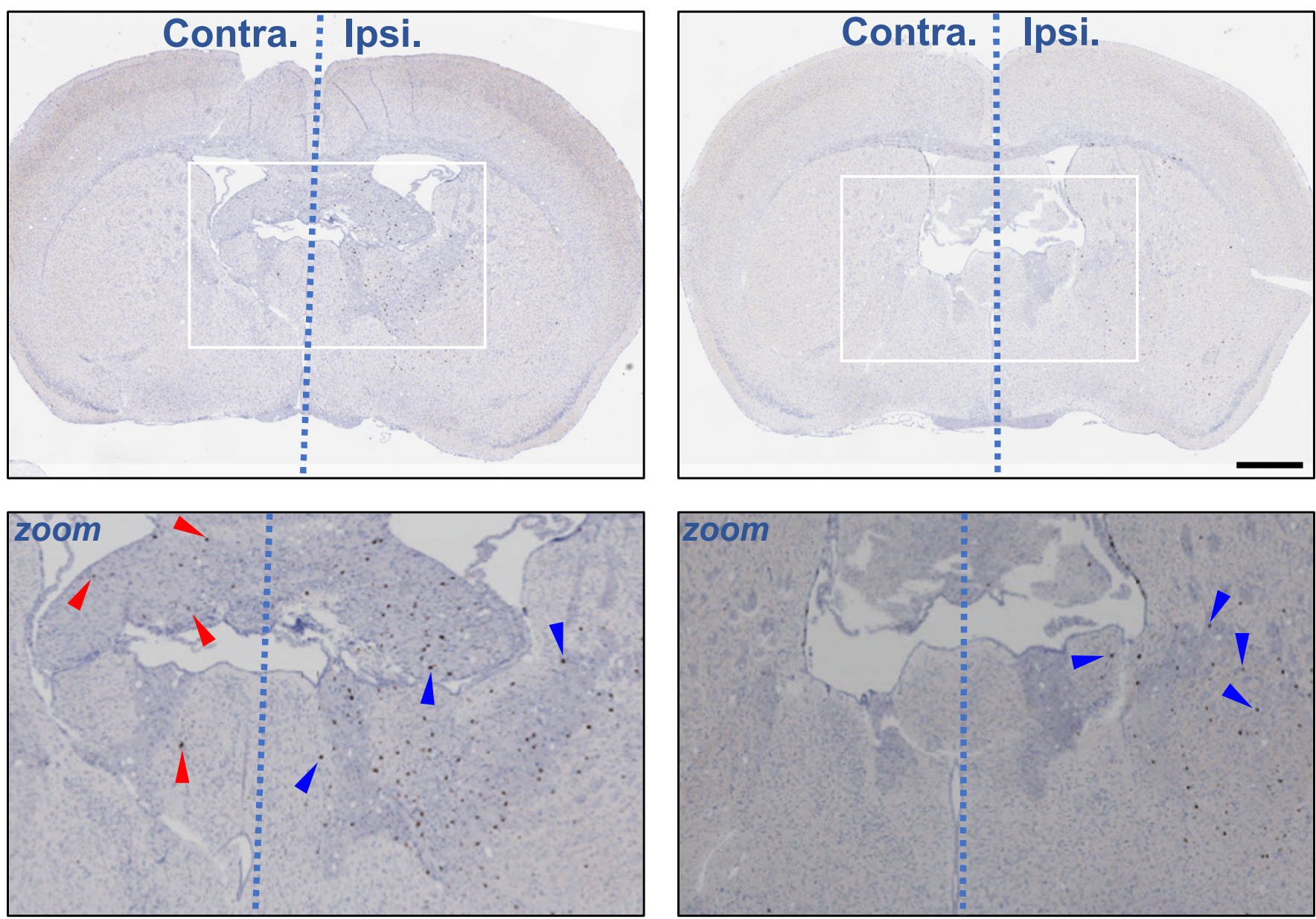

B

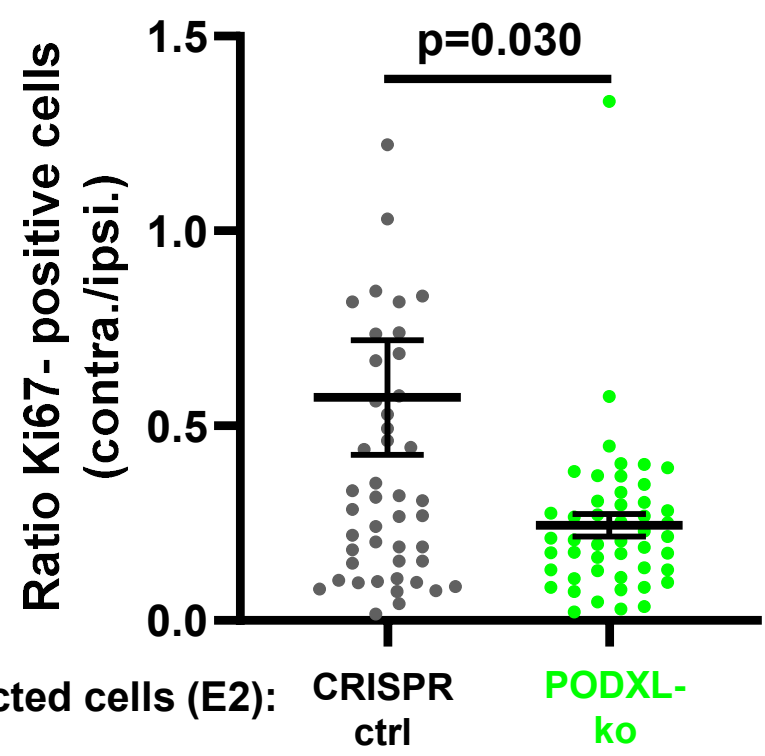




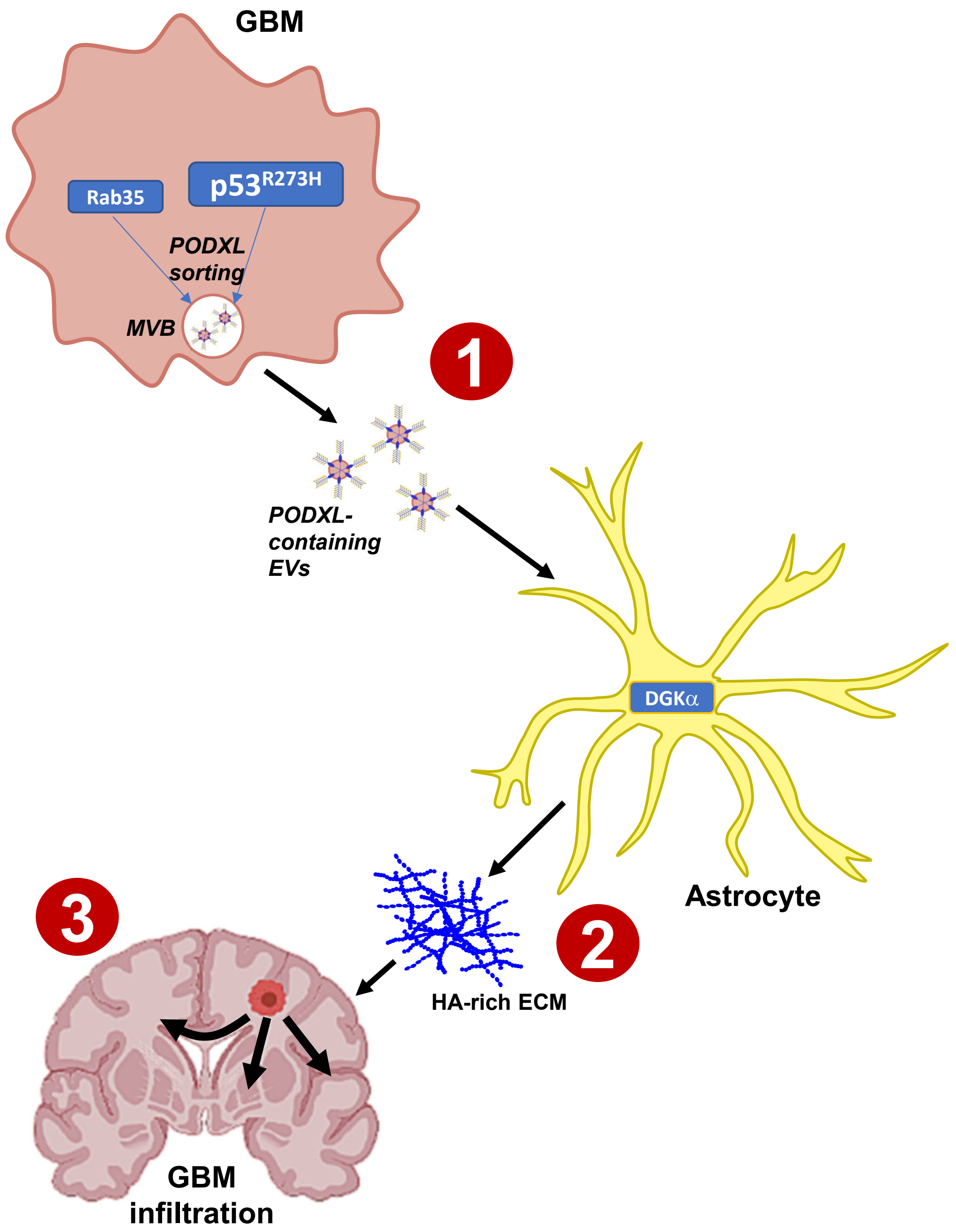

